\title{
THE COMPLEXITY OF CLASSIFYING SEPARABLE BANACH SPACES UP TO ISOMORPHISM
}

\author{
VALENTIN FERENCZI, ALAIN LOUVEAU, AND CHRISTIAN ROSENDAL
}

To Alekos Kechris, on the occasion of his 60 th birthday $^{1}$

\begin{abstract}
It is proved that the relation of isomorphism between separable Banach spaces is a complete analytic equivalence relation, i.e., that any analytic equivalence relation Borel reduces to it. Thus, separable Banach spaces up to isomorphism provide complete invariants for a great number of mathematical structures up to their corresponding notion of isomorphism. The same is shown to hold for (1) complete separable metric spaces up to uniform homeomorphism, (2) separable Banach spaces up to Lipschitz isomorphism, and (3) up to (complemented) biembeddability, (4) Polish groups up to topological isomorphism, and (5) Schauder bases up to permutative equivalence. Some of the constructions rely on methods recently developed by S. Argyros and P. Dodos.
\end{abstract}

\section{Contents}

1. Introduction 1

2. Notation and concepts of descriptive set theory 3

3. The standard Borel space of separable Banach spaces 3

4. Results 4

5. A variant of the completeness method 6

6. An $\ell_{n}$-tree basis 11

$\begin{array}{ll}\text { 7. Permutative equivalence } & 13\end{array}$

8. Uniform homeomorphism of complete separable metric spaces 14

$\begin{array}{ll}\text { 9. Isomorphism of separable Banach spaces } & 19\end{array}$

References 22

\section{INTRODUCTION}

A general mathematical problem is that of classifying one class of mathematical objects by another, that is, given some class $\mathcal{A}$, e.g., countable groups, and a corresponding notion of isomorphism one tries to find complete invariants for the

Date: June 2006.

2000 Mathematics Subject Classification. Primary: 46B03, Secondary $03 E 15$.

Key words and phrases. Isomorphism of Banach spaces, Complexity of analytic equivalence relations.

The third author is partially supported by NSF grant DMS 0556368 .

${ }^{1}$ The main result of this paper was obtained at the end of a conference held in UrbanaChampaign in honour of A.S. Kechris. We take this opportunity to thank the organisers C. W. Henson and S. Solecki for their hospitality. 
objects in $\mathcal{A}$ up to isomorphism. In other words, one tries to assign to each object in $\mathcal{A}$ some other object such that two objects in $\mathcal{A}$ have the same assignment if and only if they are isomorphic. This way of stating it is however slightly misleading, as, in general, one cannot do better than assigning isomorphism classes in some other category $\mathcal{B}$, or more precisely, on can make an assignment from $\mathcal{A}$ to $\mathcal{B}$ such that two objects in $\mathcal{A}$ are isomorphic if and only if their assignments in $\mathcal{B}$ are isomorphic. In this case, we say that we have classified the objects in $\mathcal{A}$ by the objects of $\mathcal{B}$ up to isomorphism. However, in order for this not to be completely trivial, one would like the assignment itself to be somehow calculable or explicit. The classification should not just rely on some map provided by the axiom of choice.

For a period going back at least 20 years, there has been a concentrated effort in descriptive set theory to make a coherent theory out of the notion of classification and to determine which classes of objects can properly be said to be classifiable by others. The way in which this has been done is by considering standard Borel spaces that can be considered to fully represent the classes of objects in question, and then study the corresponding notion of isomorphism as an equivalence relation on the space. As standard Borel spaces are fully classified by their cardinality, which can be either countable or $2^{\aleph_{0}}$, the perspective changes from the objects in question to the equivalence relation instead. One therefore talks of classifying equivalence relations by each other instead of the corresponding objects. If an equivalence relation is classifiable by another, one says that the former is less complex than the latter. Here is the precise definition.

Definition 1. Let $E$ and $F$ be equivalence relations on standard Borel spaces $X$ and $Y$ respectively. We say that $E$ is Borel reducible to $F$ if there is a Borel function $f: X \rightarrow Y$ such that

$$
x E y \leftrightarrow f(x) F f(y)
$$

for all $x, y \in X$. We denote this by $E \leqslant B$ and informally say that $E$ less complex than $F$. If both $E \leqslant_{B} F$ and $F \leqslant_{B} E$, then $E$ and $F$ are called Borel bireducible, written $E \sim \sim_{B} F$.

If one looks at the classes of objects that readily are considered as a standard Borel space $X$, e.g., countable combinatorial and algebraic objects, separable complete metric structures, one notices that the corresponding notion of isomorphism is most often analytic, if not Borel, seen as a subset of $X^{2}$. Because of this, and also because the structure theory of $\leqslant_{B}$ breaks down beyond the level of analytic or Borel, one has mostly only developed the theory in this context.

Classical examples of classifications that fit nicely into this theory are the classification of countable boolean algebras by compact metric spaces up to homeomorphism by Stone duality and the Orstein classification of Bernoulli automorphisms by entropy.

An easy fact, first noticed by Leo Harrington, is that among the analytic equivalence relations there is necessarily a maximum one with respect to the ordering $\leqslant_{B}$. However, for a long period no concrete example of this maximum one was found, only abstract set theoretical versions were known. This problem was solved by Louveau and Rosendal in [14, but at a certain expense. It was noticed that the definition of the Borel reducibility ordering extends ad verbatim to quasiorders, i.e., transitive and reflexive relations, and that one again has a maximum analytic quasiorder. Using a representation result that we shall come back to later, 
it was shown that, e.g., the relation of embeddability between countable graphs is a complete analytic quasiorder, i.e., that its $\leqslant{ }_{B}$-degree is maximum among analytic quasiorders. A simple argument then shows that the corresponding equivalence relation of bi-embeddability is a complete analytic equivalence relation.

A large theory has now been developed concerning analytic and Borel equivalence relations, but, of course, a main interest in this theory comes from the fact that it should provide an understanding of concrete mathematical examples. Thus, in functional analysis, much effort has been made on trying to understand the structure of Banach spaces by making inroads into the classification problem, i.e., by trying to classify separable Banach spaces up to (linear) isomorphism. Since this is obviously an immensely complicated task (exactly how immense should be clear from the main result of this paper), one hoped for a long time that one should instead be able to find simple subspaces present in every space. However, even this has turned out somewhat harder than hoped for by several bad examples of spaces due to Tsirelson [23] and, especially, Gowers and Maurey [12].

We will here show exactly how complicated the task is by showing that the relation of isomorphism between separable Banach spaces is actually complete as an analytic equivalence relation and therefore that the classification problem for separable Banach spaces is at least as complicated as almost any other classification problem of analysis.

\section{NotATION AND CONCEPTS OF DESCRIPTIVE SET THEORY}

In all of the following we will write $\omega$ for the set of natural numbers $\mathbb{N}=$ $\{0,1,2, \ldots\}$ and 2 for the two-element set $\{0,1\}$. If $A$ is a non-empty set, a tree $T$ on $A$ will be a set of finite strings $t=\left(a_{0}, a_{1}, \ldots, a_{n-1}\right) \in A^{<\omega}$ of elements of $A$, $n \geqslant 0$, containing the empty string $\emptyset$ and such that if $s \subseteq t$ and $t \in T$, then $s \in T$. Here $s \subseteq t$ denotes that $s$ is an initial segment of $t$.

A Polish space is a separable topological space whose topology is given by a complete metric. The Borel sets in a Polish space are those sets that belong to the smallest $\sigma$-algebra containing the open sets. A standard Borel space is the underlying set of a Polish space equipped with the Borel algebra. By a theorem of Kuratowski, all uncountable standard Borel spaces are Borel isomorphic with $\mathbb{R}$. An analytic or $\boldsymbol{\Sigma}_{1}^{1}$-set is a subset of a standard Borel space that is the image by a Borel function of another standard Borel space. A set is coanalytic if its complement is analytic.

A very useful way of thinking of Borel and analytic sets, which is now known as the Kuratowski-Tarski algorithm, is in terms of the quantifier complexity of their definitions. Thus, Borel sets are those that can be inductively defined by using only countable quantifiers, i.e., quantifiers over countable sets, while analytic sets are those that can be defined using countable quantifiers and a single positive instance of a quantifier over a Polish space.

\section{The standard Borel space of Separable Banach spaces}

In order to consider the class of separable Banach spaces as a standard Borel space, we take a separable metrically universal Banach space, e.g., $X=C([0,1])$ and denote by $F(X)$ the set of all its closed subsets. We equip $F(X)$ with its so

called Effros-Borel structure, which is the $\sigma$-algebra generated by the sets on the 
form

$$
\{F \in F(X) \mid F \cap U \neq \emptyset\},
$$

where $U$ varies over open subsets of $X$. Equipped with this $\sigma$-algebra, $F(X)$ becomes a standard Borel space, i.e., isomorphic as a measure space with $\mathbb{R}$ given its standard Borel algebra. It is then a standard fact, which is not hard to verify, that the subset $\mathfrak{B} \subseteq F(X)$ consisting of all the closed linear subspaces of $X$ is a Borel set in the Effros-Borel structure. So, in particular, $\mathfrak{B}$ is itself a standard Borel space, and it therefore makes sense to talk of Borel and analytic classes of separable Banach spaces, referring by this to the corresponding subset of $\mathfrak{B}$. We therefore consider $\mathfrak{B}$ as the space of separable Banach spaces. It is an empirical fact that any other way of defining this leads to equivalent results.

The same construction can be done for complete separable metric spaces. In this case, we begin with a separable complete metric space, universal for all complete separable metric spaces, for concreteness we take the Urysohn metric space $\mathbb{U}$, and then let $\mathfrak{M}$ be the standard Borel space of all its closed subsets equipped with the Effros-Borel structure. Again, we see $\mathfrak{M}$ as the space of all complete separable metric spaces. Since there is a natural inclusion $\mathfrak{B} \subseteq \mathfrak{M}$, it is reassuring to know that $\mathfrak{B}$ is a Borel subset of $\mathfrak{M}$.

We shall consider several notions of comparison between Banach spaces that all will turn out to provide analytic relations on $\mathfrak{M}$.

Definition 2. Let $\left(X, d_{X}\right)$ and $\left(Y, d_{Y}\right)$ be metric spaces. We say that

- $X$ and $Y$ are Lipschitz isomorphic if there is a bijection $f: X \rightarrow Y$ such that for some $K \geqslant 1$, we have for all $x, y$

$$
\frac{1}{K} d_{X}(x, y) \leqslant d_{Y}(f(x), f(y)) \leqslant K d_{X}(x, y) .
$$

- $X$ is Lipschitz embeddable into $Y$ if $X$ is Lipschitz isomorphic with a subset of $Y$.

- $X$ and $Y$ are uniformly homeomorphic if there is a bijection $f: X \rightarrow Y$ such that both $f$ and $f^{-1}$ are uniformly continuous.

To see that, for example, the relation of Lipschitz isomorphism on $\mathfrak{M}$ is analytic, we notice that for $X, Y \in \mathfrak{M}, X$ is Lipschitz isomorphic to $Y$ if and only if

$$
\begin{aligned}
\exists K \geqslant 1 \exists\left(x_{n}\right) \exists\left(y_{n}\right) & \left(\forall m\left(X \cap U_{m} \neq \emptyset \rightarrow \exists n x_{n} \in U_{m}\right)\right. \\
& \& \forall m\left(Y \cap U_{m} \neq \emptyset \rightarrow \exists n y_{n} \in U_{m}\right) \\
& \&\left(\forall n x_{n} \in X\right) \&\left(\forall n y_{n} \in Y\right) \\
& \left.\& \forall n, m\left(\frac{1}{K} d_{X}\left(x_{n}, x_{m}\right) \leqslant d_{Y}\left(y_{n}, y_{m}\right) \leqslant K d_{X}\left(x_{n}, x_{m}\right)\right)\right)
\end{aligned}
$$

where $\left\{U_{m}\right\}$ is an open basis for $\mathbb{U}$. In other words, $X$ and $Y$ are Lipschitz isomorphic if and only if they have Lipschitz isomorphic countable dense subsets and this condition can be expressed in an analytic manner in $\mathfrak{M}^{2}$.

\section{RESUlts}

We are now ready to explain the results of the present paper. The main idea is to combine a refinement of the completeness results of Louveau and Rosendal in 14. with a recent construction by S. Argyros and P. Dodos from [1] that was done for different though related purposes. The thrust of the Agyros-Dodos construction is 
to be able to associate to each analytic set of Schauder bases (i.e., analytic set of subsequences of the universal Pełczyński basis) a separable space that essentially only has basic sequences from the analytic set. Of course this is not quite possible (take, for example, $\left\{\ell_{1}, \ell_{2}\right\}$ ), but at least one can get a significant amount of control over the types of basic sequences present. Now the completeness results of 14, on the other hand, show exactly that certain relations related to identity (in the codes) of analytic sets are complete analytic equivalence relations, and we are therefore able to code these relations into relations between Banach spaces.

Our first result concerns the relation of permutative equivalence between Schauder bases. First, as mentioned above, we take as our standard Borel space of Schauder bases, the set $[\omega]^{\omega}$ of all infinite subsets of $\omega=\{0,1, \ldots\}$, where we identify a subset of $\omega$ with the corresponding subsequence of the universal Schauder basis constructed by Pełzcyński [18]. It was proved in [20] that the relation of equivalence between Schauder bases was a complete $\boldsymbol{K}_{\sigma}$-quasiorder, and here we prove

Theorem 3. The relation of permutative equivalence between (even unconditional) Schauder bases is a complete analytic equivalence relation.

This result gives probably the a priori simplest naturally occurring equivalence relation which is known to be analytic complete. Thus, somewhat surprisingly, the relation of isomorphism between separable Banach spaces is Borel reducible to permutative equivalence. One can actually consider this even as a sort of representation result for, e.g., separable Banach spaces. We can in a Borel manner associate to each space a basis such that two spaces are isomorphic if and only if the two bases are permutatively equivalent. If this could be done in a more informative or explicit manner than in our construction, one could really hope for an increased understanding of the isomorphism relation in terms of the more readily understandable relation of permutative equivalence, and, in fact, one could consider such a result a best positive solution to the problem of representing separable Banach spaces by bases.

We subsequently use Theorem [ to study uniform homeomorphism between complete separable metric spaces. Though we have not been able to show that this relation restricted to $\mathfrak{B}$ is complete analytic, we do get

Theorem 4. The relation of uniform homeomorphism between complete separable metric spaces is a complete analytic equivalence relation.

There is of course another perhaps more immediate relation to study on $\mathfrak{M}$, namely isometry. The situation for this relation is nevertheless slightly different, for Gao and Kechris 11] have shown that this relation is bireducible with the most complex orbit equivalence relation, $E_{G}$, induced by the continuous action of a Polish group on a Polish space. And by results of Kechris and Louveau 13, this relation is strictly less complex than a complete analytic equivalence relation. So this also means that isometry on $\mathfrak{B}$ is simpler than permutative equivalence of bases. Recently, Melleray [16] has been able to show that also restricted to $\mathfrak{B}$ is the (linear) isometry relation Borel bireducible with $E_{G}$. This should be contrasted with the result in [14] saying that the relation of (linear) isometric biembeddability on $\mathfrak{B}$ is a complete analytic equivalence relation.

The most important relation between Banach spaces is however the relation of linear isomorphism, which has turned out to be exceedingly difficult to understand, 
so much that among Banach space theorists there has even been a feeling that the category of Banach spaces might not be the right category to study, but that one instead should consider only spaces with a basis. Theorem 3 of course shows that such a restriction would not really decrease the complexity of the task, but at least the following result should be a comfort in the sense that it confirms the feeling of outmost complexity.

Theorem 5. The relations of isomorphism and Lipschitz isomorphism between separable Banach spaces are complete analytic equivalence relations.

This result is the culmination of a series of successive lower estimates of the complexity by Bossard 4, Rosendal [19], and Ferenczi-Galego 8. We also consider the corresponding quasiorders of embeddability etc. and show that these are also complete in their category.

We finally consider Banach spaces as abelian groups and notice that any continuous group isomorphism is also linear. Therefore,

Theorem 6. The relation of topological isomorphism between (abelian) Polish groups is a complete analytic equivalence relation.

Previous work on the complexity of isomorphism between groups has been exclusively on the countable discrete case. An early result of Friedman and Stanley [10] states that the relation of isomorphism between countable discrete groups is complete among all isomorphism relations between countable structures, while Thomas and Velickovic 22] prove that restricted to the class of finitely generated groups it becomes complete among all Borel equivalence relations having countable classes.

\section{A variant of the COMPleteness method}

In 14, Louveau and Rosendal established a representation result for analytic quasiorders, and used this result to prove that some $\boldsymbol{\Sigma}_{1}^{1}$ quasiorders are complete, i.e., have the property that any other $\boldsymbol{\Sigma}_{1}^{1}$ quasiorder is Borel reducible to them, and to deduce from this that certain $\boldsymbol{\Sigma}_{1}^{1}$ equivalence relations are also complete .

It was clear, from the way the completeness results were derived from the representation that the technique was flexible and could lead to improved results. This was implicitly acknowledged in 14 and stated more explicitly in 20 in the case of $\boldsymbol{K}_{\sigma}$ quasiorders, but, as at the time no essential use of it was made, the details were not spelled out. However, in the applications in the present paper, the finer versions have turned out to be crucial for our proofs and we therefore proceed to state the results precisely.

The main idea is to desymmetrise the situation, both for the relations and for the reducibility ordering. We think of a binary relation $R$ on some $X$ as the pair of relations $(R, \neg R)$, where $\neg R$ denotes the complement of $R$ in $X^{2}$. With this identification, Borel reducibility is now defined on certain kinds of pairs, and we extend it to arbitrary pairs as follows.

Definition 7. Let $\left(R_{1}, R_{2}\right)$ and $\left(S_{1}, S_{2}\right)$ be two pairs of binary relations on standard Borel spaces $X$ and $Y$ respectively. A Borel map $f: X \rightarrow Y$ is a Borel homomorphism from $\left(R_{1}, R_{2}\right)$ to $\left(S_{1}, S_{2}\right)$ if for all $x, y \in X, x R_{1} y \rightarrow f(x) S_{1} f(y)$, and $x R_{2} y \rightarrow f(x) S_{2} f(y)$. We say that $\left(R_{1}, R_{2}\right)$ is Borel hom-reducible to $\left(S_{1}, S_{2}\right)$, and write

$$
\left(R_{1}, R_{2}\right) \preccurlyeq B\left(S_{1}, S_{2}\right)
$$


if there is a Borel homomorphism from $\left(R_{1}, R_{2}\right)$ to $\left(S_{1}, S_{2}\right)$.

Borel hom-reducibility is clearly a quasi-ordering, as homomorphisms can be composed. Moreover, one has from the definitions that

$$
R \leqslant_{B} S \leftrightarrow(R, \neg R) \preccurlyeq \preccurlyeq_{B}(S, \neg S),
$$

so that using the identification above, $\preccurlyeq_{B}$ is indeed an extension of $\leqslant_{B}$.

In the sequel, we will let $\left(R_{1}, R_{2}\right) \leqslant_{B} R$ and $R \leqslant_{B}\left(R_{1}, R_{2}\right)$ stand for $\left(R_{1}, R_{2}\right) \preccurlyeq B$ $(R, \neg R)$ and $(R, \neg R) \preccurlyeq B\left(R_{1}, R_{2}\right)$, respectively.

Suppose now that we are interested in a class $\mathcal{C}$ of binary relations on standard Borel spaces, e.g., analytic quasiorders or analytic equivalence relations. We say that a pair $\left(R_{1}, R_{2}\right)$ of binary relations is $\mathcal{C}$-hard if any element of $R \in \mathcal{C}$ is Borel hom-reducible to $\left(R_{1}, R_{2}\right)$, or more precisely $R \leqslant_{B}\left(R_{1}, R_{2}\right)$. And we say that $\left(R_{1}, R_{2}\right)$ is $\mathcal{C}$-complete if it is $\mathcal{C}$-hard, and, moreover, it is Borel hom-reducible to some element of $\mathcal{C}$. In other words, if we set

$$
\mathcal{C}^{*}=\left\{\left(R_{1}, R_{2}\right) \mid \exists R \in \mathcal{C}\left(R_{1}, R_{2}\right) \leqslant B R\right\}
$$

the pair $\left(R_{1}, R_{2}\right)$ is $\mathcal{C}$-complete if it is $\preccurlyeq B_{B}$-maximum in $\mathcal{C}^{*}$. It is very easy to check that there is a $\mathcal{C}$-complete $R$, i.e., a $\leqslant_{B}$-maximum element in $\mathcal{C}$, if and only if there is a $\mathcal{C}$-complete pair $\left(R_{1}, R_{2}\right)$, and, moreover, if this happens, then the $\mathcal{C}$-complete $R$ 's are exactly the ones which, viewed as pairs, are complete.

However, the strong completeness results we will need in the sequel only rely on the following simple observation: Suppose $\left(R_{1}, R_{2}\right)$ is $\mathcal{C}$-hard and $\left(R_{1}, R_{2}\right)$ reduces to some $R \in \mathcal{C}$. Then $R$ is in fact $\mathcal{C}$-complete. This is of course an obvious fact, but will be quite handy as it will allow us to work in some cases with a more manageable pair $\left(R_{1}, R_{2}\right)$ than with a single complete relation $R$.

To give the flavour of the arguments, consider the case of $\boldsymbol{\Sigma}_{1}^{1}$ equivalence relations. Then there is a pair which is easily seen to be hard for this class: suppose we are given a coding $\alpha \mapsto A_{\alpha}$ of $\boldsymbol{\Sigma}_{1}^{1}$ subsets of say $2^{\omega}$ by elements of some Polish space $X$ (we will be more specific later on), and define binary relations $=\boldsymbol{\Sigma}_{1}^{1}, \subseteq \boldsymbol{\Sigma}_{1}^{1}, \operatorname{Disj}_{\boldsymbol{\Sigma}_{1}^{1}}$ on $X$ corresponding, "in the codes", to $=, \subseteq$, and disjointness between non-empty $\Sigma_{1}^{1}$ sets, i.e., set

$$
\begin{aligned}
\alpha=_{\Sigma_{1}^{1}} \beta \leftrightarrow A_{\alpha} & \neq \emptyset \& A_{\beta} \neq \emptyset \& A_{\alpha}=A_{\beta}, \\
\alpha \subseteq_{\Sigma_{1}^{1}} \beta \leftrightarrow A_{\alpha} & \neq \emptyset \& A_{\beta} \neq \emptyset \& A_{\alpha} \subseteq A_{\beta}, \\
\alpha \operatorname{Disj}_{\boldsymbol{\Sigma}_{1}^{1}} \beta \leftrightarrow A_{\alpha} & \neq \emptyset \& A_{\beta} \neq \emptyset \& A_{\alpha} \cap A_{\beta}=\emptyset .
\end{aligned}
$$

For any reasonable coding, the pair $\left(=\boldsymbol{\Sigma}_{1}^{1}, \operatorname{Disj}_{\boldsymbol{\Sigma}_{1}^{1}}\right)$ is hard for the class of $\boldsymbol{\Sigma}_{1}^{1}$ equivalence relations. For if $E$ is such a relation, which without loss of generality we can view as defined on $2^{\omega}$, one can associate to $x \in 2^{\omega}$ a code for its equivalence class $[x]_{E}$ in a continuous way, and this gives a homomorphism from $E$ to the pair $\left(=\boldsymbol{\Sigma}_{1}^{1}\right.$, Disj $\left._{\boldsymbol{\Sigma}_{1}^{1}}\right)$. We do not know if this pair is complete for analytic equivalence relations, hence if it can be used to obtain completeness results. But the basic representation of [14] allows to replace it by a complete pair.

Let us first recall this result:

Theorem 8 (A. Louveau and C. Rosendal 14]). Let $R \subseteq 2^{\omega} \times 2^{\omega}$ be a $\boldsymbol{\Sigma}_{1}^{1} q u a$ siorder. Then there exists a tree $T$ on $2 \times 2 \times \omega$ with the following properties

(1) $x R y \leftrightarrow \exists \alpha \in \omega^{\omega} \forall n\left(\left.x\right|_{n},\left.y\right|_{n},\left.\alpha\right|_{n}\right) \in T$,

(2) if $(u, v, s) \in T$ and $s \leqslant t$, then $(u, v, t) \in T$, 
(3) for all $(u, s) \in(2 \times \omega)^{<\omega}$, we have $(u, u, s) \in T$,

(4) if $(u, v, s) \in T$ and $(v, w, t) \in T$, then $(u, w, s+t) \in T$.

In the statement of the above theorem, if $s$ is a finite sequence and $|s|$ denotes its length, we let for sequences $s$ and $t, s \leqslant t$ mean that $|s|=|t|$ and for all $i<|s|, s(i) \leqslant t(i)$. And for $s, t$ of the same length, we let $(s+t)(i)=s(i)+t(i)$.

Let $\mathfrak{T}$ be the class of non-empty normal trees on $2 \times \omega$, i.e., trees $T$ with $(\emptyset, \emptyset) \in T$ and such that whenever $(u, s) \in T$ and $s \leqslant t$, then also $(u, t) \in T$. Viewed as a subset of $2^{(2 \times \omega)^{<\omega}}$, it is closed, hence a (compact) Polish space.

We view each normal tree $T$ as coding the $\boldsymbol{\Sigma}_{1}^{1}$ set

$$
A(T)=\left\{\alpha \in 2^{\omega} \mid \exists \beta \in \omega^{\omega} \forall n\left(\left.\alpha\right|_{n},\left.\beta\right|_{n}\right) \in T\right\} .
$$

As is well known, any $\boldsymbol{\Sigma}_{1}^{1}$ subset of $2^{\omega}$ is on the form $A(T)$ for some $T$, so we really have a coding.

Definition 9. We define the following binary relations on $\mathfrak{T}$. For $S, T \in \mathfrak{T}$ we let

$$
\begin{aligned}
S & \leqslant \boldsymbol{\Sigma}_{1}^{1} T \\
S \equiv_{\boldsymbol{\Sigma}_{1}^{1}} T & \leftrightarrow S \leqslant \omega_{\boldsymbol{\Sigma}_{1}^{1}} T \& T(u, s)\left((u, s) \in S \rightarrow\left(u, s+\left.\alpha\right|_{|s|}\right) \in T\right), \\
S \mathbb{\Sigma}_{\boldsymbol{\Sigma}_{1}^{1}} T & \leftrightarrow A(S) \nsubseteq A(T), \\
S \operatorname{Disj}_{\boldsymbol{\Sigma}_{1}^{1}} T & \leftrightarrow A(S) \neq \emptyset \& A(T) \neq \emptyset \& A(S) \cap A(T)=\emptyset, \\
S \neq_{\boldsymbol{\Sigma}_{1}^{1}} T & \leftrightarrow A(S) \neq A(T) .
\end{aligned}
$$

Theorem 10. (i) The pair $\left(\leqslant_{\boldsymbol{\Sigma}_{1}^{1}}, \mathbb{}_{\boldsymbol{\Sigma}_{1}^{1}}\right)$ is complete for the class $\mathcal{C}_{\mathrm{qo}}$ of analytic quasiorders.

(ii) The pair $\left(\equiv_{\boldsymbol{\Sigma}_{1}^{1}}, \operatorname{Disj}_{\boldsymbol{\Sigma}_{1}^{1}}\right)$ is complete for the class $\mathcal{C}_{\mathrm{eq}}$ of analytic equivalence relations, and hence, a fortiori, the pair $\left(\equiv_{\boldsymbol{\Sigma}_{1}^{1}}, \neq_{\boldsymbol{\Sigma}_{1}^{1}}\right)$ is complete for $\mathcal{C}_{\mathrm{eq}}$ too.

Proof. Note first that $\leqslant_{\boldsymbol{\Sigma}_{1}^{1}}$ is an analytic quasiorder, and $\equiv_{\boldsymbol{\Sigma}_{1}^{1}}$ an analytic equivalence relation, with trivially

$$
\left(\leqslant \boldsymbol{\Sigma}_{1}^{1}, \mathbb{\Sigma}_{\boldsymbol{\Sigma}_{1}^{1}}\right) \leqslant{ }_{B} \leqslant \boldsymbol{\Sigma}_{1}^{1}
$$

and

$$
\left(\equiv_{\boldsymbol{\Sigma}_{1}^{1}}, \operatorname{Disj}_{\boldsymbol{\Sigma}_{1}^{1}}\right) \preccurlyeq B\left(\equiv_{\boldsymbol{\Sigma}_{1}^{1}}, \neq_{\boldsymbol{\Sigma}_{1}^{1}}\right) \leqslant_{B} \equiv_{\boldsymbol{\Sigma}_{1}^{1}}
$$

via the identity map, so that it is enough to prove that the pairs are hard for their respective classes.

For part (i), let $R$ be a $\Sigma_{1}^{1}$ quasiorder on some Polish space $X$. Embedding $X$ into $2^{\omega}$ in a Borel way, we may assume $R$ is defined on $2^{\omega}$. Let then $T$ be the tree given by theorem 8 and define a continuous map $f: 2^{\omega} \rightarrow \mathfrak{T}$ by

$$
f(x)=\left\{(u, s) \in(2 \times \omega)^{<\omega} \mid\left(u,\left.x\right|_{|u|}, s\right) \in T\right\} .
$$

We claim this map works. First, each $f(x)$ is indeed a non empty normal tree by properties (2) and (3) of $T$. Also, by property (1), $A(f(x))=\left\{y \in 2^{\mathbb{N}} y R x\right\}$, so that if $\neg x R y$, we get $x \in A(f(x))$ but $x \notin A(f(y))$, whence $f(x) \mathbb{E}_{\boldsymbol{\Sigma}_{1}^{1}} f(y)$. Conversely suppose $x R y$. Then by property (1) of $T$ there is some $\alpha \in \omega^{\omega}$ with $\left(\left.x\right|_{n},\left.y\right|_{n},\left.\alpha\right|_{n}\right) \in$ $T$ for all $n$. But then this $\alpha$ witnesses $f(x) \leqslant \Sigma_{1}^{1} f(y)$, for if $(u, s) \in f(x)$, i.e., if $\left(u,\left.x\right|_{|u|}, s\right) \in T$, we get from property (4) of $T$ that $\left(u,\left.y\right|_{|u|}, s+\left.\alpha\right|_{|u|}\right) \in T$, as $\left(\left.x\right|_{|u|},\left.y\right|_{|u|},\left.\alpha\right|_{|u|}\right) \in T$. So $\left(u, s+\left.\alpha\right|_{|u|}\right) \in f(y)$, as desired. This proves (i).

To prove (ii), we again assume that the Polish space is $2^{\omega}$. As an equivalence relation $E$ is in particular a quasiorder, we can apply part (i), and get a continuous 
map $f: 2^{\omega} \rightarrow \mathfrak{T}$ such that $x E y \rightarrow f(x) \leqslant \boldsymbol{\Sigma}_{1}^{1} f(y)$ and $A(f(x))=[x]_{E}$. But then trivially $f$ hom-reduces $(E, \neg E)$ to $\left(\equiv_{\boldsymbol{\Sigma}_{1}^{1}}, \operatorname{Disj}_{\boldsymbol{\Sigma}_{1}^{1}}\right)$, as wanted.

The previous result is conceptually the simplest one. Unfortunately, we will need in the sequel a slight improvement of the last statement, obtained by restricting the domains of the relations to pruned normal trees, which makes things messier.

Recall that a non empty tree is pruned if any sequence in it admits a strict extension that is still in it.

Let $\mathfrak{T}_{\mathrm{pr}}$ be the $G_{\delta}$ subset of $\mathfrak{T}$ consisting of the non-empty pruned normal trees, and denote by $\leqslant_{\Sigma_{1}^{1}}^{\mathrm{pr}}, \equiv_{\boldsymbol{\Sigma}_{1}^{1}}^{\mathrm{pr}}, \mathbb{E}_{\boldsymbol{\Sigma}_{1}^{1}}^{\mathrm{pr}}$ and $\neq_{\boldsymbol{\Sigma}_{1}^{1}}^{\mathrm{pr}}$ the restrictions to $\mathfrak{T}_{\mathrm{pr}}$ of the corresponding relations.

Theorem 11. (i) The pair $\left(\leqslant_{\boldsymbol{\Sigma}_{1}^{1}}^{\mathrm{pr}}, \nsubseteq_{\boldsymbol{\Sigma}_{1}^{1}}^{\mathrm{pr}}\right)$ is complete for the class $\mathcal{C}_{\mathrm{qo}}$.

(ii) The pair $\left(\equiv_{\boldsymbol{\Sigma}_{1}^{1}}^{\mathrm{pr}}, \neq_{\boldsymbol{\Sigma}_{1}^{1}}^{\mathrm{pr}}\right)$ is complete for the class $\mathcal{C}_{\mathrm{eq}}$.

Proof. (ii) follows from (i) as before, and by the previous theorem, it is enough to prove that $\left(\equiv_{\boldsymbol{\Sigma}_{1}^{1}}, \neq_{\boldsymbol{\Sigma}_{1}^{1}}\right) \preccurlyeq B\left(\equiv_{\boldsymbol{\Sigma}_{1}^{1}}^{\mathrm{pr}}, \neq_{\boldsymbol{\Sigma}_{1}^{1}}^{\mathrm{pr}}\right)$.

Let $T$ be a non-empty normal tree. We define a tree $T^{*}$ as follows: for each $(u, s)$ in $T$ of length say $n$, put in $T^{*}$ all sequences $\left(u^{\prime}, s^{\prime}\right)$ of length $\geqslant 2 n$ which satisfy

(a) $\forall i<n u^{\prime}(2 i)=u(i) \& s^{\prime}(2 i)=s(i)$,

(b) $\forall i<n u^{\prime}(2 i+1)=0$, and (c) $\forall i \geqslant 2 n u^{\prime}(i)=1$,

together with their initial segments. Easily $T^{*}$ is still normal, and is now pruned as any sequence in $T^{*}$ can be extended using (c). So this defines a continuous map from $\mathfrak{T}$ to $\mathfrak{T}_{\text {pr }}$, and it is enough to check it is the homomorphism we want. For each $\alpha \in \omega^{\omega}$, set $\alpha^{*}(2 i)=\alpha(i)$ and $\alpha^{*}(2 i+1)=0$. Then one checks easily using (a) and (b) that if $\alpha$ is a witness that $S \leqslant \boldsymbol{\Sigma}_{1}^{1} T$, then $\alpha^{*}$ witnesses that $S^{*} \leqslant_{\Sigma_{1}^{1}}^{\mathrm{pr}} T^{*}$. Let also $D_{1} \subseteq 2^{\mathbb{N}}$ be the countable set of eventually 1 sequences, and for $u \in 2^{n}$, let $\alpha_{u}(2 i)=u(i)$ and $\alpha_{u}(2 i+1)=0$ for $i<n$, and $\alpha_{u}(i)=1$ for $i \geqslant 2 n$. Then easily from (a), (b), and (c) one gets that

$$
A\left(T^{*}\right)=\left\{\alpha^{*} \mid \alpha \in A(T)\right\} \cup\left\{\alpha_{u} \mid \exists s(u, s) \in T\right\} .
$$

From this we get that $A(S) \nsubseteq A(T)$ implies that $A\left(S^{*}\right) \nsubseteq A\left(T^{*}\right)$ as desired.

Note that one does not have necessarily $A\left(S^{*}\right) \cap A\left(T^{*}\right)=\emptyset$ when $A(S) \cap A(T)=\emptyset$. Still we could define $S \operatorname{Disj}_{\boldsymbol{\Sigma}_{1}^{1}}^{\mathrm{pr}} T$ for $S, T \in \mathfrak{T}_{\mathrm{pr}}$ by

$$
A\left(S^{*}\right) \backslash D_{1} \neq \emptyset \& A\left(T^{*}\right) \backslash D_{1} \neq \emptyset \& A\left(S^{*}\right) \cap A\left(T^{*}\right) \subseteq D_{1}
$$

and get a slight improvement on part (ii), as this last relation is both smaller and descriptively simpler than $\neq_{\boldsymbol{\Sigma}_{1}^{1}}^{\mathrm{pr}}$.

The interesting part in this result is the following: if one wants to prove that a certain analytic equivalence relation $E$ is complete by providing a reduction from normal (pruned) trees, one needs to show that if the two trees code the same analytic set in a strong sense, namely that there is a uniform $\alpha$ that can translate between the codes, then the images are $E$-equivalent. But on the other hand, for the negative direction one only needs to consider trees that really code different analytic sets and show that their images are $E$-inequivalent. In the applications, we will construct objects from normal trees that "realise only the types" given by the analytic set corresponding to the tree. For example, in the case of separable 
Banach spaces, we shall construct from a normal tree coding an analytic subset of $] 1,2$ [ a Banach space whose only $\ell_{p}$ subspaces are exactly $\ell_{2}$ plus those given by the analytic set. Thus if two normal trees are $\neq_{\boldsymbol{\Sigma}_{1}^{1}}$ related, they have different $\ell_{p}$ subspaces and are hence non-isomorphic. A similar line of thinking in terms of extreme pairs of quasiorders is also present in Camerlo [5].

Before we go to Banach spaces, let us illustrate the previous discussion with a natural example of a $\mathcal{C}_{\mathrm{qo}}$-complete pair which could potentially be of use elsewhere. In analogy with the case of separable Banach spaces, where the class of $\ell_{p}$ subspaces of a space will turn out to be sufficient to separate non-isomorphic spaces, we search for simple types of objects in a certain category and then associate with each object its "spectrum" consisting of the simple types embeddable into it.

Let $\mathfrak{A}$ be the class of combinatorial trees on $\mathbb{N}$, i.e., acyclic, connected, symmetric relations on $\mathbb{N}$ and let $\mathfrak{A}_{\mathrm{f}}$ be the subclass of trees of finite valency. For each $T \in \mathfrak{A}$, we let $\sigma(T)$ be the spectrum of $T$, which is the set of all $S \in \mathfrak{A}_{\mathrm{f}}$ that embed into $T$. We then let $\sqsubseteq$ be the relation of embeddability between combinatorial trees and put $S \subseteq \sigma T$ if $\sigma(S) \subseteq \sigma(T)$. Using a simple modification of the construction in 14. showing that $\sqsubseteq$ is a complete analytic quasiorder one can show that

Proposition 12. The pair $\left(\sqsubseteq, \nsubseteq_{\sigma}\right)$ is $\mathcal{C}_{\mathrm{qo}}$-complete.

In view of this proposition, it would be interesting to find combinatorial realisations of the complete analytic equivalence relation. However, as is well-known, no Borel class of countable model-theoretical structures provides an isomorphism relation which is complex enough. Instead, one should look for other notions of isomorphism. A potential candidate would be the relation of quasi-isometry between countable graphs. Here two graphs $R$ and $S$ on the vertex set $\mathbb{N}$ are said to be quasi-isometric if there is a function $\phi: \mathbb{N} \rightarrow \mathbb{N}$ and numbers $K, N$ and $L$ such that for all $n, m$

$$
\begin{aligned}
& d_{R}(n, m) \leqslant K d_{S}(\phi(n), \phi(m))+N, \\
& d_{S}(\phi(n), \phi(m)) \leqslant K d_{R}(n, m)+N,
\end{aligned}
$$

and

$$
\forall k \exists l d_{S}(k, \phi(l)) \leqslant L .
$$

S. Thomas [21] has recently proved that this relation, when restricted to locally finite graphs, is Borel bireducible with the complete $\boldsymbol{K}_{\sigma}$-equivalence relation.

To end up this section, let us discuss another situation where it is possible to get a nice complete pair (although we have no application for it). It is the case of orbit equivalence relations for Borel actions of Polish groups.

Fix a Polish group $G$. If $X$ is a standard Borel space and $\alpha: G \times X \rightarrow X$ is a Borel action of $G$ on $X$, one defines the associated $\boldsymbol{\Sigma}_{1}^{1}$ orbit equivalence $E_{G}^{X}$ by

$$
x E_{G}^{X} y \leftrightarrow \exists g \in G \alpha(g, x)=y .
$$

We let $\mathcal{C}_{G}$ be the class of all such orbit equivalence relations. By a result of BeckerKechris [2], it is the same class, up to Borel isomorphism, as the class of orbit equivalences corresponding to continuous actions of $G$ on Polish spaces $X$.

Becker and Kechris also proved that there is a complete element in $\mathcal{C}_{G}$. We now provide a complete pair for it (which gives a somewhat different complete element).

First, fix some universal Polish space $X_{0}$, like the Urysohn space or $\mathbb{R}^{\omega}$, with the property that any Polish space is homeomorphic to a closed subspace of it. Let $Z$ 
be the standard Borel space of non-empty closed subsets of $X_{0} \times G$, equipped with the Effros-Borel structure. Define an action $(g, F) \mapsto g . F$ of $G$ on $Z$ by setting $g . F=\{(x, g h) \mid(x, h) \in F\}$. It is easy to check that this action is Borel, and hence the associated $E_{G}^{Z}$ is in $\mathcal{C}_{G}$.

For $F$ in $Z$, set $A(F)=\left\{x \in X_{0} \mid \exists g \in G(x, g) \in F\right\}$, and define $\operatorname{Disj}_{G}$ on $Z$ by $F \operatorname{Disj}_{G} F^{\prime} \leftrightarrow A(F) \cap A\left(F^{\prime}\right)=\emptyset$.

Theorem 13. The pair $\left(E_{G}^{Z}, \operatorname{Disj}_{G}\right)$ is complete for the class $\mathcal{C}_{G}$ (and hence $E_{G}^{Z}$ is complete too).

Proof. As $E_{G}^{Z}$ is in $\mathcal{C}_{G}$, we only have to check that $\left(E_{G}^{Z}, \operatorname{Disj}_{G}\right)$ is $\mathcal{C}_{G}$-hard. And by the result of Becker and Kechris quoted above, we only have to consider Polish spaces $X$ and continuous actions $\alpha: G \times X \rightarrow X$. View $X$ as a closed subset of $X_{0}$ and associate to each $x \in X$ the element $F_{x} \in Z$ defined by

$$
F_{x}=\left\{(y, g) \in X_{0} \times G \mid y \in X \& \alpha(y, g)=x\right\} .
$$

Note that $F_{x}$ is non-empty as $\left(x, 1_{G}\right) \in F_{x}$, and one can check, using the continuity of $\alpha$, that the map $x \mapsto F_{x}$ is Borel. One easily also checks that $F_{\alpha(g, x)}=g \cdot F_{x}$, so that if $x E_{G}^{X} y$, then $F_{x} E_{G}^{Z} F_{y}$. And finally $A\left(F_{x}\right)$ is just the orbit of $x$ for the action $\alpha$, hence if $\neg x E_{G}^{X} y, A\left(F_{x}\right) \cap A\left(F_{y}\right)=\emptyset$, as desired.

\section{AN $\ell_{p}$-TREE BASIS}

We define in this section the construction of a basic sequence from a tree on $2 \times \omega$. This will prove to be fundamental in our later proofs. We begin by choosing a Cantor set of $p$ 's in the interval ]1,2[ in the following fashion. The set is given by a Cantor scheme $\left(I_{u}\right)_{u \in 2^{<\omega}}$ of non-empty closed subintervals $\left.I_{u} \subseteq\right] 1,2[$ such that the following holds.

(1) $I_{u 0} \cup I_{u 1} \subseteq I_{u}$,

(2) $\max I_{u 0}<\min I_{u 1}$,

(3) $I_{u 0}$ contains the left endpoint of $I_{u}$,

(4) $I_{u 1}$ contains the right endpoint of $I_{u}$,

(5) the standard unit vector bases of $\ell_{\min I_{u}}^{|u|}$ and $\ell_{\max I_{u}}^{|u|}$ are 2-equivalent, i.e., $\frac{|u|^{1 / \min I_{u}}}{|u|^{1 / \max I_{u}}} \leqslant 2$, for all $u \neq \emptyset$.

If now $\alpha \in 2^{\mathbb{N}}$, we denote by $p_{\alpha}$ the unique point in $\bigcap_{u \subseteq \alpha} I_{u}$. Then

$$
\left.\alpha \in 2^{\mathbb{N}} \mapsto p_{\alpha} \in\right] 1,2[
$$

is an orderpreserving homeomorphism between $2^{\mathbb{N}}$ with the lexicographical ordering and a compact subset of $] 1,2[$.

In the following we denote by $\mathrm{T}$ the complete normal tree $(2 \times \omega)^{<\omega}$. As always we identify the elements of $\mathrm{T}$ with the pairs $t=(u, s) \in 2^{<\omega} \times \omega^{<\omega}$ such that $|u|=|s|$. A segment $\mathfrak{s}$ of $\mathrm{T}$ is just a set on the form $\mathfrak{s}=\left\{t \in \mathrm{T} \mid t_{0} \subseteq t \subseteq t_{1}\right\}$ for some $t_{0}, t_{1} \in \mathrm{T}$.

We now let $\mathcal{V}=c_{00}(\mathbf{T})$ be the vector space with basis $\left(e_{t}\right)_{t \in \mathbf{T}}$. For each segment

$$
\mathfrak{s}=\left\{\left(u_{0}, s_{0}\right) \subsetneq\left(u_{1}, s_{1}\right) \subsetneq \ldots \subsetneq\left(u_{n}, s_{n}\right)\right\}
$$

of $\mathrm{T}$, we define a norm $\|\cdot\|_{\mathfrak{s}}$ on $\mathcal{V}$ as follows

$$
\left\|\sum_{t \in \mathrm{T}} \lambda_{t} e_{t}\right\|_{\mathfrak{s}}=\sup _{m \leqslant n}\left\|\left(\lambda_{\left(u_{0}, s_{0}\right)}, \lambda_{\left(u_{1}, s_{1}\right)}, \ldots, \lambda_{\left(u_{m}, s_{m}\right)}\right)\right\|_{\min I_{u_{m}}} .
$$


We notice that for $m \leqslant n$, we have $u_{m} \subseteq u_{n}$ and so $I_{u_{n}} \subseteq I_{u_{m}}$, whence by condition (5)

$$
\begin{aligned}
& \left\|\left(\lambda_{\left(u_{0}, s_{0}\right)}, \lambda_{\left(u_{1}, s_{1}\right)}, \ldots, \lambda_{\left(u_{m}, s_{m}\right)}\right)\right\|_{\min I_{u_{m}}} \\
\leqslant & \left\|\left(\lambda_{\left(u_{0}, s_{0}\right)}, \lambda_{\left(u_{1}, s_{1}\right)}, \ldots, \lambda_{\left(u_{m}, s_{m}\right)}\right)\right\|_{\min I_{u_{n}}} \\
\leqslant & 2\left\|\left(\lambda_{\left(u_{0}, s_{0}\right)}, \lambda_{\left(u_{1}, s_{1}\right)}, \ldots, \lambda_{\left(u_{m}, s_{m}\right)}, \ldots, \lambda_{\left(u_{n}, s_{n}\right)}\right)\right\|_{\min I_{u_{n}}} .
\end{aligned}
$$

Thus, if $\sigma=(\alpha, \beta) \in[\mathrm{T}]$ is a branch of $\mathrm{T}$ containing the segment $\mathfrak{s}$, then $p_{\alpha} \in I_{u_{n}}$, and thus

$$
\left\|\sum_{t \in \mathfrak{s}} \lambda_{t} e_{t}\right\|_{p_{\alpha}} \leqslant\left\|\sum_{t \in \mathfrak{s}} \lambda_{t} e_{t}\right\|_{\mathfrak{s}}=\left\|\sum_{t \subseteq \sigma} \lambda_{t} e_{t}\right\|_{\mathfrak{s}} \leqslant 2\left\|\sum_{t \in \mathfrak{s}} \lambda_{t} e_{t}\right\|_{p_{\alpha}} \leqslant 2\left\|\sum_{t \subseteq \sigma} \lambda_{t} e_{t}\right\|_{p_{\alpha}}
$$

Finally, we define the norm $\|\cdot\|$ on $\mathcal{V}$ by

$$
\left\|\sum_{t \in \mathbf{T}} \lambda_{t} e_{t}\right\|=\sup \left\{\left(\sum_{i=1}^{l}\left\|\sum_{t \in \mathfrak{s}_{i}} \lambda_{t} e_{t}\right\|_{\mathfrak{s}_{i}}^{2}\right)^{\frac{1}{2}} \mid\left(\mathfrak{s}_{i}\right)_{i=1}^{l} \text { are incomparable segments of } \mathrm{T}\right\}
$$

and denote by $\mathcal{T}_{2}$ the completion of $\mathcal{V}$ under this norm. The space $\mathcal{T}_{2}$ is what is called an $\ell_{2}$-Baire sum in [1] and will play a universality role in the following. We notice first that $\left(e_{t}\right)_{t \in \mathrm{T}}$ is a suppression unconditional basis for $\mathcal{T}_{2}$, i.e., the projection onto any subsequence has norm 1 , and we need therefore not concern ourselves with any particular enumeration of it in ordertype $\omega$. For any branch $\sigma=(\alpha, \beta) \in[\mathrm{T}]$, we denote by $X_{\sigma}$ the closed subspace of $\mathcal{T}_{2}$ generated by the vectors $\left(e_{t}\right)_{t \subseteq \sigma}$. Then $\left(e_{t}\right)_{t \subseteq \sigma}=\left(e_{\sigma \mid n}\right)_{n<\omega}$ is a suppression unconditional Schauder basis for $X_{\sigma}$, which by inequality (11) is 2-equivalent with the standard unit vector basis of $\ell_{p_{\alpha}}$.

We should note the following thing about the segment norm. Assume

$$
\mathfrak{s}=\left\{\left(u_{0}, s_{0}\right) \subsetneq\left(u_{1}, s_{1}\right) \subsetneq \ldots \subsetneq\left(u_{n}, s_{n}\right)\right\}
$$

and

$$
\mathfrak{s}^{\prime}=\left\{\left(u_{0}, s_{0}^{\prime}\right) \subsetneq\left(u_{1}, s_{1}^{\prime}\right) \subsetneq \ldots \subsetneq\left(u_{n}, s_{n}^{\prime}\right)\right\}
$$

are two segments of $\mathrm{T}$ whose first coordinates coincide, then

$$
\left\|\lambda_{0} e_{\left(u_{0}, s_{0}\right)}+\ldots+\lambda_{n} e_{\left(u_{n}, s_{n}\right)}\right\|_{\mathfrak{s}}=\left\|\lambda_{0} e_{\left(u_{0}, s_{0}^{\prime}\right)}+\ldots+\lambda_{n} e_{\left(u_{n}, s_{n}^{\prime}\right)}\right\|_{\mathfrak{s}^{\prime}} .
$$

This will allow us to prove the following lemma.

Lemma 14. Let $S, T$ be subtrees of $\mathrm{T}$ and $\phi: S \rightarrow T$ an isomorphism of trees preserving the first coordinates, i.e., for all $(u, s) \in S$ there is some $s^{\prime}$ such that $\phi(u, s)=\left(u, s^{\prime}\right)$. Then the map

$$
M_{\phi}: e_{(u, s)} \mapsto e_{\phi(u, s)}
$$

extends to a surjective linear isometry from the space $Z_{S}=\left[e_{t}\right]_{t \in S} \subseteq \mathcal{T}_{2}$ onto $Z_{T}=\left[e_{t}\right]_{t \in T} \subseteq \mathcal{T}_{2}$.

Proof. By symmetry it suffices to prove that for any finite linear combination $x$ of $\left(e_{t}\right)_{t \in S}$ we have $\|x\| \leqslant\left\|M_{\phi}(x)\right\|$.

So fix incomparable segments $\left(\mathfrak{s}_{i}\right)_{i=1}^{l}$ of $\mathrm{T}$ and consider the estimation

$$
\left(\sum_{i=1}^{l}\left\|\sum_{t \in \mathfrak{s}_{i}} \lambda_{t} e_{t}\right\|_{\mathfrak{s}_{i}}^{2}\right)^{\frac{1}{2}} \leqslant\|x\|
$$


As the support of $x$ is completely contained in $S$, we can, by projecting onto suitable initial segments, suppose, without changing the lower estimate of $\|x\|$, that each $\mathfrak{s}_{i}$ is completely contained within $S$. But then

$$
\left(\sum_{i=1}^{l}\left\|\sum_{t \in \mathfrak{s}_{i}} \lambda_{t} e_{t}\right\|_{\mathfrak{s}_{i}}^{2}\right)^{\frac{1}{2}}=\left(\sum_{i=1}^{l}\left\|\sum_{t \in \mathfrak{s}_{i}} \lambda_{t} e_{\phi(t)}\right\|_{\phi\left[\mathfrak{s}_{i}\right]}^{2}\right)^{\frac{1}{2}} \leqslant\left\|M_{\phi}(x)\right\| .
$$

So by taking suprema we see that $\|x\| \leqslant\left\|M_{\phi}(x)\right\|$.

\section{Permutative equivalence}

We are now in a position to show that the relation of permutative equivalence between (suppression unconditional) basic sequences is a complete analytic equivalence relation.

We let $\mathcal{U B S}$ denote the standard Borel space of unconditional basic sequences, i.e., $\mathcal{U B S}$ can be chosen to be the set of subsequences of the universal unconditional basic sequence $\left(u_{n}\right)$ of Pełzcyński (see [18]). Denote by $\left(x_{i}\right) \approx_{p}\left(y_{i}\right)$ the fact that the two bases $\left(x_{i}\right)$ and $\left(y_{i}\right)$ in $\mathcal{U B S}$ are permutatively equivalent, i.e., for some permutation $f$ of $\mathbb{N},\left(x_{i}\right) \approx\left(y_{f(i)}\right)$. We recall that, as was first noticed by Mityagin [17, unconditional basic sequences satisfy the Schröder-Bernstein principle, i.e., if $\left(x_{i}\right)$ and $\left(y_{i}\right)$ are normalised unconditional basic sequences and $f, g: \mathbb{N} \rightarrow \mathbb{N}$ injections such that $\left(x_{i}\right) \approx\left(y_{f(i)}\right)$ and $\left(y_{i}\right) \approx\left(x_{g(i)}\right)$, then $\left(x_{i}\right)$ and $\left(y_{i}\right)$ are permutatively equivalent. This is easily seen to follow from the proof of the Schröder-Bernstein Theorem.

Theorem 15. The relation of permutative equivalence, $\approx_{p}$, between unconditional basic sequences is a complete analytic equivalence relation.

Proof. We shall reduce the pair $\left(\equiv_{\boldsymbol{\Sigma}_{1}^{1}}, \neq_{\boldsymbol{\Sigma}_{1}^{1}}\right)$ between pruned normal trees on $2 \times \omega$ to $\approx_{p}$. The reduction $\phi$ is the obvious one given by

$$
\phi: S \mapsto\left(e_{t}\right)_{t \in S},
$$

where $\left(e_{t}\right)_{t \in S}$ is enumerated in ordertype $\omega$ in some canonical way. Here $\left(e_{t}\right)_{t \in \mathrm{T}}$ is the canonical basis for the space $\mathcal{T}_{2}$. Since $\left(e_{t}\right)_{t \in S}$ is suppression unconditional, it remains a basic sequence any way we enumerate it.

Suppose first that $S$ and $T$ are pruned normal trees on $2 \times \omega$ such that $S \equiv_{\boldsymbol{\Sigma}_{1}^{1}} T$ as witnessed by some $\alpha \in \omega^{\omega}$. Then, by Lemma 14] $e_{(u, s)} \mapsto e_{\left(u, s+\left.\alpha\right|_{|s|}\right)}$ restricts to an isometric embedding of $\left[e_{t}\right]_{t \in S}$ into $\left[e_{t}\right]_{t \in T}$ and also to an isometric embedding of $\left[e_{t}\right]_{t \in T}$ into $\left[e_{t}\right]_{t \in S}$. In particular, the unconditional bases $\left(e_{t}\right)_{t \in S}$ and $\left(e_{t}\right)_{t \in T}$ are equivalent to subsequences of each other and hence permutatively equivalent.

On the other hand, if $S \neq_{\boldsymbol{\Sigma}_{1}^{1}} T$, we can find some $\alpha \in 2^{\omega}$ such that $\alpha \in$ $\operatorname{proj}[S] \backslash \operatorname{proj}[T]$. Take some $\beta \in \omega^{\omega}$ such that $(\alpha, \beta) \in[S]$, and notice then that $\left(e_{(\alpha|n, \beta| n)}\right)_{n}$ is equivalent with the unit vector basis in $\ell_{p_{\alpha}}$. We claim that there is no subsequence of $\left(e_{t}\right)_{t \in T}$ equivalent with $\ell_{p_{\alpha}}$. To see this, notice that if $\left(e_{t}\right)_{t \in A}$ was any subsequence of $\left(e_{t}\right)_{t \in T}$, then by Ramsey's theorem we could find some infinite subset $B \subseteq A$ such that either $B \subseteq\{(\gamma|n, \delta| n) \mid n \in \mathbb{N}\}$ for some $(\gamma, \delta) \in[T]$ or $B$ is an antichain in $T$.

In the first case, $\left(e_{t}\right)_{t \in B}$ is equivalent with a subsequence of the unit vector basis of $\ell_{p_{\gamma}}$ and hence, as $p_{\gamma} \neq p_{\alpha}$, is not equivalent with $\ell_{p_{\alpha}}$, and in the latter case, by construction of $\mathcal{T}_{2},\left(e_{t}\right)_{t \in B}$ is equivalent with $\ell_{2}$, which is not equivalent with 
$\ell_{p_{\alpha}}$ either. Thus $S \neq_{\boldsymbol{\Sigma}_{1}^{1}} T \Rightarrow\left(e_{t}\right)_{t \in S} \not_{p}\left(e_{t}\right)_{t \in T}$. This finishes the proof of the reduction.

We easily see from the above construction that we also reduce the pair

$$
\left(\leqslant \Sigma_{1}^{1}, \mathbb{\Sigma}_{\boldsymbol{\Sigma}_{1}^{1}}\right)
$$

to the relation of being permutatively equivalent with a subsequence between unconditional basic sequences. And thus

Theorem 16. The relation between unconditional basic sequences of being permutatively equivalent with a subsequence is a complete analytic quasiorder.

The are several related results concerning equivalence of basic sequences. For example, Ferenczi and Rosendal show in 9 that a basic sequence is either subsymmetric, i.e., equivalent with all its subsequences, or the relation $E_{0}$ Borel reduces to equivalence between its subsequences. Also Rosendal [20] shows that the relation of equivalence between basic sequences is Borel bireducible with a complete $K_{\sigma}$ equivalence relation. And finally, Ferenczi [7] proves that if $\left(e_{i}\right)$ is an unconditional basic sequence, then either $E_{0}$ Borel reduces to the relation of permutative equivalence between the normalised blockbases of $\left(e_{i}\right)$, or, for some $\ell_{p}$ or $c_{0}$, any normalised blockbasis has a subsequence equivalent with this $\ell_{p}$ or $c_{0}$.

\section{UNIFORM HOMEOMORPHISM OF COMPLETE SEPARABLE METRIC SPACES}

We now intend to show that the relation of uniform homeomorphism between complete separable metric spaces is a complete analytic equivalence relation. This will be done by reducing the relation of permutative equivalence between unconditional basic sequences to it. Let us first remark that uniform homeomorphism is indeed analytic. To see this, notice that if $\left(X, d_{X}\right)$ and $\left(Y, d_{Y}\right)$ are two complete separable metric spaces, then they are uniformly homeomorphic if and only if they have countable dense subsets $D_{X}$ and $D_{Y}$ that are uniformly homeomorphic. For any uniform homeomorphism between $D_{X}$ and $D_{Y}$ will preserve Cauchy sequences in both directions and hence extend to a uniform homeomorphism between $X$ and $Y$. But the relation of uniform homeomorphism between countable metric spaces is easily seen to be analytic, whence this extends to all complete separable metric spaces.

This argument clearly does not extend to the relation of homeomorphism between complete separable metric spaces (or more naturally to the class of Polish topological spaces). A priori this relation is not analytic but only $\boldsymbol{\Sigma}_{2}^{1}$, but, as we shall see, it is $\boldsymbol{\Sigma}_{1}^{1}$-hard as an equivalence relation. It is natural to ask the following.

Question 17. Is the relation of homeomorphism between Polish spaces, i.e., closed subspaces of $\mathbb{R}^{\mathbb{N}}$, a complete $\Sigma_{2}^{1}$ equivalence relation?

In the following, we fix a normalised bimonotone unconditional basic sequence $\left(e_{n}\right)_{n \in N}$ in a Banach space, where $N$ is an unordered infinite countable set, and we let $X$ be the closed subspace generated by the sequence $\left(e_{n}\right)_{n \in N}$, and denote by $d_{X}$ the metric on $X$.

A type is a non-empty finite non-decreasing sequence of strictly positive rational numbers. If $t=\left(\lambda_{1}, \ldots, \lambda_{n}\right)$ is a type, we say that a vector $x \in X$ has type $t$ when $x$ can be written $x=\sum_{i=1}^{m} \lambda_{i} e_{\sigma(i)}$, for some injection $\sigma$ of $\{1, \ldots, m\}$ into $N$. Since $\left(e_{n}\right)$ is a basis, it is clear that each vector of $X$ has at most one type. We enumerate 
the set of types as $\left(t_{n}\right)_{n \in \mathbb{N}}$, with $t_{1}=(1)$, and we let $T_{n}$ be the set of vectors of $X$ of type $t_{n}$; in particular $T_{1}$ is the set of the unit vectors of the basis $\left(e_{n}\right)_{n \in N}$.

Lemma 18. For any $n \in \mathbb{N}$, there exists $\delta_{n}>0$ such that the set $T_{n}$ is $\delta_{n}$-separated.

Proof. Write $t_{n}=\left(\lambda_{1}, \ldots, \lambda_{m}\right)$, let $\lambda_{0}=0$, and let

$$
\delta_{n}=\min \left(\left\{\left|\lambda_{i}-\lambda_{j}\right| \mid 0 \leqslant i, j \leqslant m\right\} \cap \mathbb{R}_{+}^{*}\right) .
$$

If $x, y \in T_{n}$ are distinct, then there exists $k \in N$ such that $p_{k}(x) \neq p_{k}(y)$, where $p_{k}$ denotes the (norm 1) projection onto $\left[e_{k}\right]$. Since $p_{k}(x)$ (respectively $\left.p_{k}(y)\right)$ is equal to $\lambda e_{k}$ for some $\lambda \in\left\{\lambda_{i} \mid 0 \leqslant i \leqslant m\right\}$, it follows that $\left|p_{k}(x)-p_{k}(y)\right| \geqslant \delta_{n}$, and therefore that $\|x-y\| \geqslant\left|p_{k}(x-y)\right| \geqslant \delta_{n}$.

We now describe how to build a Polish space $P(X)$ by implanting on $X$ various elementary metric spaces in order to rigidify its topological structure.

For any $n \in \mathbb{N}$, let $H_{n}$ be a fixed metric space with a special point $0_{n}$, which is the union of $n$ isometric copies of $[0,1]$, each of which has $0_{n}$ as endpoint, and which intersect only in $0_{n}$. Now for any $n \in \mathbb{N}$ and $x \in X$ of type $t_{n}$, we let $H(x)$ be an isometric copy of $H_{n}$ in which we denote the special point corresponding to $0_{n}$ by $x$, and we denote by $d_{x}$ the metric on $H(x)$. We also denote $H^{0}(x)=H(x) \backslash\{x\}$.

We then let $P(X)$ be the amalgamation of $X$ with all $H(x)$, for $n \in \mathbb{N}$ and $x \in T_{n}$, each $H(x)$ being amalgamated with $P(X)$ in $x$. This means that

$$
P(X)=X \sqcup\left(\bigsqcup_{n \in \mathbb{N}} \bigsqcup_{x \in T_{n}} H^{0}(x)\right),
$$

where the metric $d$ on $P(X)$ is defined as follows, for $y, z$ in $P(X)$ :

- if $y$ and $z$ both belong to $X$, then $d(y, z)=d_{X}(y, z)$,

- if $y$ and $z$ both belong to some $H^{0}(x)$, then $d(y, z)=d_{x}(y, z)$,

- if $y \in X$ and $z$ belongs to some $H^{0}(x)$, then $d(y, z)=d_{X}(y, x)+d_{x}(x, z)$,

- if $y$ belongs to some $H^{0}(x)$ and $z$ belongs to some $H^{0}\left(x^{\prime}\right)$, with $x \neq x^{\prime}$, then $d(y, z)=d_{x}(y, x)+d_{X}\left(x, x^{\prime}\right)+d_{x^{\prime}}\left(x^{\prime}, z\right)$.

The set $R=\bigcup_{n \in \mathbb{N}} T_{n} \subset P(X)$ is called the set of roots in $P(X)$, and the set $H=\bigcup_{x \in R} H^{0}(x) \subset P(X)$ is called the hair in $P(X)$. We have the following fact:

Lemma 19. The space $P(X)$ is separable and complete metric.

Proof. $P(X)$ is obviously separable. If $\left(y_{k}\right)_{k \in \mathbb{N}}$ is a Cauchy sequence in $P(X)$, and $y_{k}$ belongs to $X$ for all $k \in \mathbb{N}$, then $\left(y_{k}\right)_{k}$ converges in $X$; therefore we may assume that $y_{k}$ belongs to the hair for all $k \in \mathbb{N}$. If there is a fixed $x \in X$ such that $y_{k}$ belongs to $H^{0}(x)$ for all $k \in \mathbb{N}$, then $\left(y_{k}\right)_{k}$ converges in $H^{0}(x) \cup\{x\}$ by completeness of $H(x)$; therefore we may assume that there is a sequence $\left(x_{k}\right)_{k \in \mathbb{N}}$ of pairwise distinct points of $X$ such that $y_{k} \in H^{0}\left(x_{k}\right)$ for all $k \in \mathbb{N}$. Then for all $j, k$ in $\mathbb{N}$,

$$
d\left(y_{j}, y_{k}\right)=d\left(y_{j}, x_{j}\right)+d\left(x_{j}, x_{k}\right)+d\left(x_{k}, y_{k}\right),
$$

and we deduce that $\left(x_{k}\right)_{k}$ is a Cauchy sequence in $X$ and that $\left(d\left(x_{k}, y_{k}\right)\right)_{k}$ converges to 0 ; therefore the sequence $\left(y_{k}\right)_{k}$ converges to some $x$ in $X$.

Proposition 20. Let $\left(e_{n}\right)_{n \in N}$ and $\left(e_{n}^{\prime}\right)_{n \in N^{\prime}}$ be normalised bimonotone unconditional basic sequences and let $X=\left[e_{n}, n \in N\right], X^{\prime}=\left[e_{n}^{\prime}, n \in N^{\prime}\right]$. Then any homeomorphism between $P(X)$ and $P\left(X^{\prime}\right)$ takes $X$ onto $X^{\prime}$, the hair in $P(X)$ onto the hair in $P\left(X^{\prime}\right)$, the set of roots in $P(X)$ onto the set of roots in $P\left(X^{\prime}\right)$, and for each $n \in \mathbb{N}$, the set of type $t_{n}$ vectors of $X$ onto the set of type $t_{n}$ vectors of $X^{\prime}$. 
Proof. Indeed $H$ is the set of points in $P(X)$ which admit an open neighborhood homeomorphic to $[0,1[$ or $] 0,1[$, and $X=P(X) \backslash H$. Also, define an implant as a maximal subset of $H$ homeomorphic to [0,1[, and given $x \in X$, say that an implant $h$ is attached to $x$ if $x$ is adherent to $h$. It is then clear that a point $x$ in $P(X)$ is a root if and only if some implant is attached to it, and that $x$ is a point of type $t_{n}$, for $n \in \mathbb{N}$, if and only if exactly $n$ implants are attached to $x$.

Proposition 21. Let $\left(e_{n}\right)_{n \in N}$ and $\left(e_{n}^{\prime}\right)_{n \in N^{\prime}}$ be normalised bimonotone unconditional basic sequences and let $X=\left[e_{n}, n \in N\right], X^{\prime}=\left[e_{n}^{\prime}, n \in N^{\prime}\right]$. Let $T$ be a homeomorphism between $P(X)$ and $P\left(X^{\prime}\right)$. Then there exists a bijection $\sigma$ between $N$ and $N^{\prime}$ such that for any finite subset $I$ of $N$, any sequence $\left(\lambda_{i}\right)_{i \in I}$ of non-negative real numbers,

$$
T\left(\sum_{n \in I} \lambda_{n} e_{n}\right)=\sum_{n \in I} \lambda_{n} e_{\sigma(n)}^{\prime} .
$$

Proof. Since $T$ maps type (1) points of $P(X)$ onto type (1) points of $P\left(X^{\prime}\right)$, there exists a bijection $\sigma$ between $N$ and $N^{\prime}$ such that $T\left(e_{n}\right)=e_{\sigma(n)}^{\prime}$ for all $n \in N$. By continuity of $T$, it is then enough to prove by induction on $|I|$ that for any finite subset $I$ of $N$, and for any sequence $\left(\lambda_{n}\right)_{n \in I}$ of pairwise distinct positive rationals,

$$
T\left(\sum_{n \in I} \lambda_{n} e_{n}\right)=\sum_{n \in I} \lambda_{n} e_{\sigma(n)}^{\prime} .
$$

For any $n \in \mathbb{N}$, any $\lambda \in \mathbb{Q}^{+*}, T\left(\lambda e_{n}\right)$ has type $(\lambda)$, therefore $T\left(\lambda e_{n}\right)=\lambda e_{k_{n}(\lambda)}^{\prime}$ for some $k_{n}(\lambda)$ in $N^{\prime}$. By continuity, $e_{k_{n}(\lambda)}^{\prime}=T\left(\lambda e_{n}\right) / \lambda$ is constant on $\mathbb{Q}^{+*}$ and equal to $e_{k_{n}(1)}^{\prime}=T\left(e_{n}\right)=e_{\sigma(n)}^{\prime}$. Therefore

$$
T\left(\lambda e_{n}\right)=\lambda e_{\sigma(n)}^{\prime}, \forall \lambda \in \mathbb{Q}^{+*} .
$$

Let now $I \subset N$ be finite, with $|I| \geqslant 2$. Let $\Delta$ be the open subset of $\left(\mathbb{Q}^{+*}\right)^{I}$ defined by

$$
\Delta=\left\{\left(\lambda_{n}\right)_{n \in I}: \forall n \neq p, \lambda_{n} \neq \lambda_{p}\right\} .
$$

For $\lambda=\left(\lambda_{n}\right)_{n \in I} \in \Delta$, let $x(\lambda)=\sum_{n \in I} \lambda_{n} e_{n}$. Then $T(x(\lambda))$ has the same type as $x(\lambda)$ and therefore may be written (uniquely) in the form

$$
T(x(\lambda))=\sum_{n \in I} \lambda_{n} e_{k_{n}(\lambda)}^{\prime},
$$

with $k_{n}(\lambda) \in N^{\prime}$ for each $n \in I$.

We prove that $k_{n}(\lambda)$ is locally constant on $\Delta$, for all $n \in I$. Fix indeed $\lambda \in \Delta$, then for any $\mu=\left(\mu_{n}\right)_{n \in I}$ in a neighborhood $V$ of $\lambda$ in $\Delta$, we have

$$
\begin{aligned}
& \left\|\sum_{n \in I} \lambda_{n} e_{k_{n}(\lambda)}^{\prime}-\sum_{n \in I} \lambda_{n} e_{k_{n}(\mu)}^{\prime}\right\| \\
\leqslant & \left\|\sum_{n \in I} \lambda_{n} e_{k_{n}(\lambda)}^{\prime}-\sum_{n \in I} \mu_{n} e_{k_{n}(\mu)}^{\prime}\right\|+\left\|\sum_{n \in I}\left(\lambda_{n}-\mu_{n}\right) e_{k_{n}(\mu)}^{\prime}\right\| \\
\leqslant & \|T(x(\lambda))-T(x(\mu))\|+\sum_{n \in I}\left|\lambda_{n}-\mu_{n}\right| .
\end{aligned}
$$

Therefore if $V$ is small enough,

$$
\left\|\sum_{n \in I} \lambda_{n} e_{k_{n}(\lambda)}^{\prime}-\sum_{n \in I} \lambda_{n} e_{k_{n}(\mu)}^{\prime}\right\|<\delta, \forall \mu \in V
$$


where $\delta$ is such that the set of points of $X$ of same type as $x$ is $\delta$-separated (Lemma 18). Therefore,

$$
\sum_{n \in I} \lambda_{n} e_{k_{n}(\lambda)}^{\prime}=\sum_{n \in I} \lambda_{n} e_{k_{n}(\mu)}^{\prime}, \forall \mu \in V
$$

and since the $\lambda_{n}$ 's for $n$ in $I$ are pairwise distinct,

$$
k_{n}(\lambda)=k_{n}(\mu), \forall n \in I, \forall \mu \in V .
$$

We deduce from this fact that for all $n$ in $I, k_{n}$ is constant on each connected component of $\Delta$.

Fix now $\lambda \in \Delta$, let $C(\lambda)$ be the connected component of $\Delta$ containing $\lambda$, and let $\left(k_{n}\right)_{n \in I} \in\left(N^{\prime}\right)^{I}$ be such that

$$
T(x(\lambda))=\sum_{n \in I} \lambda_{n} e_{k_{n}}^{\prime}
$$

Let $n_{0} \in I$ be such that $\lambda_{n_{0}}=\min _{n \in I} \lambda_{n}$. For any $\left.t \in\right] 0, \lambda_{n_{0}}$ [, the element of $\Delta$ associated to $\sum_{n \in I, n \neq n_{0}} \lambda_{n} e_{n}+t e_{n_{0}}$ is in $C(\lambda)$, therefore

$$
T\left(\sum_{n \in I, n \neq n_{0}} \lambda_{n} e_{n}+t e_{n_{0}}\right)=\sum_{n \in I, n \neq n_{0}} \lambda_{n} e_{k_{n}}^{\prime}+t e_{k_{n_{0}}}^{\prime} .
$$

When $t$ converges to 0 , we obtain that

$$
\begin{aligned}
\sum_{n \in I, n \neq n_{0}} \lambda_{n} e_{k_{n}}^{\prime} & =T\left(\sum_{n \in I, n \neq n_{0}} \lambda_{n} e_{n}\right) \\
& =\sum_{n \in I, n \neq n_{0}} \lambda_{n} e_{\sigma(n)}^{\prime},
\end{aligned}
$$

by the induction hypothesis. Since the $\lambda_{n}$ 's, $n \in I$, are pairwise distinct, it follows that $\forall n \in I \backslash\left\{n_{0}\right\}, k_{n}=\sigma(n)$.

Let now $n_{1} \in I$ be such that $\lambda_{n_{1}}=\min _{n \neq n_{0}} \lambda_{n}$ and let $\lambda^{\text {sym }} \in \Delta$ be defined by $\lambda_{n}^{\text {sym }}=\lambda_{n}, \forall n \notin\left\{n_{0}, n_{1}\right\}, \lambda_{n_{0}}^{\text {sym }}=\lambda_{n_{1}}$, and $\lambda_{n_{1}}^{\text {sym }}=\lambda_{n_{0}}$. There exists $\left(l_{n}\right)_{n \in I} \in$ $\left(N^{\prime}\right)^{I}$ such that for any $\mu=\left(\mu_{n}\right)_{n \in I}$ in $C\left(\lambda^{\text {sym }}\right)$,

$$
T\left(\sum_{n \in I} \mu_{n} e_{n}\right)=\sum_{n \in I} \mu_{n} e_{l_{n}}^{\prime},
$$

and the same reasoning as above gives us that

$$
l_{n}=\sigma(n), \forall n \in I, n \neq n_{1} .
$$

Now

$$
\begin{aligned}
T\left(\sum_{n \neq n_{0}} \lambda_{n} e_{n}+\lambda_{n_{1}} e_{n_{0}}\right) & =\lim _{t \rightarrow\left(\lambda_{n_{1}}\right)^{-}} T\left(\sum_{n \neq n_{0}} \lambda_{n} e_{n}+t e_{n_{0}}\right) \\
& =\sum_{n \neq n_{0}} \lambda_{n} e_{k_{n}}^{\prime}+\lambda_{n_{1}} e_{k_{n_{0}}}^{\prime},
\end{aligned}
$$

since the element of $\Delta$ associated to $\sum_{n \neq n_{0}} \lambda_{n} e_{n}+t e_{n_{0}}$ is in $C(\lambda)$ for each $t$ in $\left[\lambda_{n_{0}}, \lambda_{n_{1}}[\right.$. Also,

$$
\begin{gathered}
T\left(\sum_{n \neq n_{0}} \lambda_{n} e_{n}+\lambda_{n_{1}} e_{n_{0}}\right)=\lim _{t \rightarrow\left(\lambda_{n_{1}}\right)^{-}} T\left(\sum_{n \neq n_{1}} \lambda_{n}^{s y m} e_{n}+t e_{n_{1}}\right) \\
=\sum_{n \neq n_{1}} \lambda_{n}^{s y m} e_{l_{n}}^{\prime}+\lambda_{n_{1}} e_{l_{n_{1}}}^{\prime}=\sum_{n \neq n_{0}} \lambda_{n} e_{l_{n}}^{\prime}+\lambda_{n_{1}} e_{l_{n_{0}}}^{\prime},
\end{gathered}
$$


since the element of $\Delta$ associated to $\sum_{n \neq n_{1}} \lambda_{n}^{s y m} e_{n}+t e_{n_{1}}$ is in $C\left(\lambda^{s y m}\right)$ for each $t \in\left[\lambda_{n_{0}}, \lambda_{n_{1}}[\right.$. Therefore

$$
\sum_{n \neq n_{0}} \lambda_{n} e_{k_{n}}^{\prime}+\lambda_{n_{1}} e_{k_{n_{0}}}^{\prime}=\sum_{n \neq n_{0}} \lambda_{n} e_{l_{n}}^{\prime}+\lambda_{n_{1}} e_{l_{n_{0}}}^{\prime}
$$

from which it follows that

$$
\left\{k_{n_{0}}, k_{n_{1}}\right\}=\left\{l_{n_{0}}, l_{n_{1}}\right\} .
$$

Since $k_{n_{1}}=\sigma\left(n_{1}\right)$ and $l_{n_{0}}=\sigma\left(n_{0}\right)$, we deduce that $k_{n_{0}}=\sigma\left(n_{0}\right)$.

We have finally proved that $k_{n}=\sigma(n), \forall n \in I$, and therefore

$$
T\left(\sum_{n \in I} \lambda_{n} e_{n}\right)=\sum_{n \in I} \lambda_{n} e_{\sigma(n)}^{\prime}, \forall\left(\lambda_{n}\right)_{n \in I} \in \Delta .
$$

Theorem 22. The pair (Lipschitz isomorphism, non-homeomorphism) restricted to the class of complete separable metric spaces is hard for analytic equivalence relations, and thus, the relation of uniform homeomorphism between complete separable metric spaces is a complete analytic equivalence relation.

Proof. We show that the relation of permutative equivalence between subsequences of the universal unconditional sequence of Pełczyński $\left(u_{n}\right)_{n \in \mathbb{N}}$ is reducible to the pair (Lipschitz isomorphism, non-homeomorphism) restricted to the class of complete separable metric spaces. Up to equivalent renorming, we may assume that $\left(u_{n}\right)_{n \in \mathbb{N}}$ is bimonotone.

For $N$ an infinite subset of $\mathbb{N}$, we define

$$
\alpha(N)=P\left(\left[u_{n}, n \in N\right]\right) .
$$

For any $N, \alpha(N)$ is canonically isometric to a closed subset of $P\left(\left[u_{n}, n \in \mathbb{N}\right]\right)$. In this setting, the map $\alpha$ is clearly Borel. Furthermore, whenever $\left(u_{n}\right)_{n \in N}$ and $\left(u_{n}\right)_{n \in N^{\prime}}$ are permutatively equivalent, there is a natural Lipschitz isomorphism between $\alpha(N)$ and $\alpha\left(N^{\prime}\right)$.

Conversely, if $T$ is a homeomorphism between $\alpha(N)$ and $\alpha\left(N^{\prime}\right)$, then by Proposition 21 there exists a bijection $\sigma$ between $N$ and $N^{\prime}$ such that for any finite subset $I$ of $N$, for any sequence $\left(\lambda_{n}\right)_{n \in I}$ of non-negative reals,

$$
T\left(\sum_{n \in I} \lambda_{n} u_{n}\right)=\sum_{n \in I} \lambda_{n} u_{\sigma(n)}
$$

It follows that $\left(u_{n}\right)_{n \in N} \approx_{p}\left(u_{n}\right)_{n \in N^{\prime}}$. Indeed, let $\left(\lambda_{n}\right)_{n} \in \mathbb{R}^{N}$ be such that $\sum_{n \in N} \lambda_{n} u_{n}$ converges, then $\sum_{n \in N}\left|\lambda_{n}\right| u_{n}$ converges by unconditionality, and therefore

$$
\sum_{n \in N}\left|\lambda_{n}\right| u_{\sigma(n)}=T\left(\sum_{n \in N}\left|\lambda_{n}\right| u_{n}\right)
$$

converges, so $\sum_{n \in \mathbb{N}} \lambda_{n} u_{\sigma(n)}$ converges, again by unconditionality. Conversely,

$$
\sum_{n \in N} \lambda_{n} u_{n}
$$

converges whenever

$$
\sum_{n \in N} \lambda_{n} u_{\sigma(n)}
$$

converges. We deduce that $\left(u_{n}\right)_{n \in N}$ is equivalent to $\left(u_{\sigma(n)}\right)_{n \in N}$. 
Unfortunately, we have not been able to replace our spaces $P(X)$ by Banach spaces and thus the following problem remains open.

Problem 23. What is the complexity with respect to $\leqslant_{B}$ of the relation of uniform homeomorphism between separable Banach spaces? In particular, is it analytic complete?

The corresponding quasiorder of uniform homeomorphic embeddability has also been studied in the form of homeomorphic embeddability between compact metric spaces. A series of results by Louveau-Rosendal 14, Marcone-Rosendal 15, and culminating in Camerlo [5, show that the relation of continuous embeddability between dendrites all of whose branching points have order three is a complete analytic quasiorder.

\section{ISOMORPHISM OF SEPARABLE BANACH SPACES}

In order to prove our main result that isomorphism of separable Banach spaces is complete, the simple construction of the spaces $Z_{S}$ does not seem to suffice. For example, it is known (see, e.g., [1]) that the space $\mathcal{T}_{2}$ contains a copy of $c_{0}$ and therefore the control over the subspaces present is presumably not good enough. Instead, we shall use the Davis, Figiel, Johnson, and Pełczyński [6 interpolation method and the results proved in [1 to avoid certain subspaces.

Suppose $S$ is a pruned subtree of T. We denote by $Z_{S}$ the closed subspace of $\mathcal{T}_{2}$ spanned by $\left(e_{t}\right)_{t \in S}$. The latter is still a suppression unconditional basis for $Z_{S}$.

In order to obtain a better control of the subspaces present, we shall now replace $Z_{S}$ with an interpolate that eliminates some vectors whose support is too much in between several different branches.

Definition 24. Let $W_{S}$ be the convex hull in $Z_{S}$ of the set $\bigcup_{\sigma \in[S]} B_{X_{\sigma}}$ and let for each $n \geqslant 0, C_{S}^{n}$ be the convex set $2^{n} W_{S}+2^{-n} B_{Z_{S}}$. As $W$ is a bounded set, we can for each $n$ define an equivalent norm on $Z_{S}$ by the taking the gauge of $C_{S}^{n}$

$$
\|x\|_{S}^{n}:=\inf \left(\lambda \mid \frac{x}{\lambda} \in C_{S}^{n}\right) .
$$

Our first lemma shows that the $n$ 'th norm of a vector does not depend on the ambient space.

Lemma 25. Let $S$ and $T$ be two pruned trees and $x \in \mathcal{V}$ a finitely supported vector belonging to both $Z_{S}$ and $Z_{T}$. Then for every $n$,

$$
\|x\|_{S}^{n}=\|x\|_{T}^{n} .
$$

Proof. Obviously, by symmetry, it is enough to prove that $\|x\|_{S}^{n} \geqslant\|x\|_{T}^{n}$. So suppose $\lambda>0$ is such that $\frac{x}{\lambda} \in C_{S}^{n}=2^{n} W_{S}+2^{-n} B_{Z_{S}}$. Then we can find a finite number of branches $\sigma_{1}, \ldots, \sigma_{m} \in[S]$, vectors $y_{i} \in B_{X_{\sigma_{i}}}$, scalars $r_{1}, \ldots, r_{m}>0$, and $z \in B_{Z_{S}}$ such that $\sum_{i} r_{i}=1$ and

$$
\frac{x}{\lambda}=2^{n}\left(r_{1} y_{1}+\ldots+r_{m} y_{m}\right)+2^{-n} z
$$

Since $x \in Z_{T}$ and has finite support, we can choose a finite number of branches $\chi_{1}, \ldots, \chi_{k} \in[T]$ such that $\operatorname{support}(x) \subseteq \chi_{1} \cup \ldots \cup \chi_{k}$. Let $R$ be the pruned tree whose branches are $\chi_{1}, \ldots, \chi_{k}$ and let $P_{R}$ be the canonical projection of $\mathcal{T}_{2}$ onto $\left[e_{t}\right]_{t \in R}$. As $\left(e_{t}\right)_{t \in \mathrm{T}}$ is suppression unconditional, $\left\|P_{R}\right\|=1$. Thus, $P_{R}(z) \in B_{Z_{T}}$ 
and, as the $y_{i}$ belong to subspaces spanned by branches, for each $i=1, \ldots, m$ there is some $1 \leqslant i^{\prime} \leqslant k$, such that $P_{R}\left(y_{i}\right) \in B_{X_{\chi_{i^{\prime}}}}$. Therefore,

$$
\begin{aligned}
\frac{x}{\lambda} & =P_{R}\left(\frac{x}{\lambda}\right) \\
& =P_{R}\left(2^{n}\left(r_{1} y_{1}+\ldots+r_{m} y_{m}\right)+2^{-n} z\right) \\
& =2^{n}\left(r_{1} P_{R}\left(y_{1}\right)+\ldots+r_{m} P_{R}\left(y_{m}\right)\right)+2^{-n} P_{R}(z) \\
& \in 2^{n} W_{T}+2^{-n} B_{Z_{T}}=C_{T}^{n} .
\end{aligned}
$$

And hence,

$$
\|x\|_{S}^{n}=\inf \left(\lambda \mid \frac{x}{\lambda} \in C_{S}^{n}\right) \geqslant \inf \left(\lambda \mid \frac{x}{\lambda} \in C_{T}^{n}\right)=\|x\|_{T}^{n}
$$

Lemma 26. Suppose $\phi: S \rightarrow T$ is an isomorphism of pruned subtrees of $\mathrm{T}$ satisfying $\phi(u, s)=\left(u, s^{\prime}\right)$, i.e., $\phi$ preserves the first coordinate of every element of $S$. Then for every $n$, the mapping

$$
M_{\phi}: e_{(u, s)} \mapsto e_{\phi(u, s)}
$$

extends (uniquely) to a surjective linear isometry from $\left(Z_{S},\|\cdot\|_{S}^{n}\right)$ onto $\left(Z_{T},\|\cdot\|_{T}^{n}\right)$.

Proof. By symmetry it is again enough to show that for any finitely supported vector $x \in Z_{S}$ we have $\|x\|_{S}^{n} \geqslant\left\|M_{\phi}(x)\right\|_{T}^{n}$. So suppose $\lambda>0$ is such that $\frac{x}{\lambda} \in C_{S}^{n}=$ $2^{n} W_{S}+2^{-n} B_{Z_{S}}$, and find a finite number of branches $\sigma_{1}, \ldots, \sigma_{m} \in[S]$, vectors $y_{i} \in B_{X_{\sigma_{i}}}$, scalars $r_{1}, \ldots, r_{m}>0$, and $z \in B_{Z_{S}}$ such that $\sum_{i} r_{i}=1$ and

$$
\frac{x}{\lambda}=2^{n}\left(r_{1} y_{1}+\ldots+r_{m} y_{m}\right)+2^{-n} z
$$

But then by Lemma 14 $M_{\phi}\left(y_{i}\right) \in B_{X_{\phi\left[\sigma_{i}\right]}}$ for each $i$, while, as $M_{\phi}$ is an isometry from $\left(Z_{S},\|\cdot\|\right)$ to $\left(Z_{T},\|\cdot\|\right)$, also $M_{\phi}(z) \in B_{Z_{T}}$. Hence, $\frac{M_{\phi}(x)}{\lambda} \in C_{T}^{n}$ and $\|x\|_{S}^{n} \geqslant$ $\left\|M_{\phi}(x)\right\|_{T}^{n}$.

Combining the two preceding lemmas we have

Lemma 27. Suppose $\phi: S \rightarrow T$ is an embedding of pruned subtrees of T satisfying $\phi(u, s)=\left(u, s^{\prime}\right)$, i.e., $\phi$ preserves the first coordinate of every element of $S$. Then for every $n$, the mapping

$$
M_{\phi}: e_{(u, s)} \mapsto e_{\phi(u, s)}
$$

extends to a linear isometry from $\left(Z_{S},\|\cdot\|_{S}^{n}\right)$ into $\left(Z_{T},\|\cdot\|_{T}^{n}\right)$.

Definition 28. Let $S$ be a pruned subtree of $\mathrm{T}$ and let $\ell_{2}\left(Z_{S},\|\cdot\|_{S}^{n}\right)$ be the $\ell_{2}$-sum of the sequence of spaces $\left(Z_{S},\|\cdot\|_{S}^{n}\right)_{n}$. We denote by $\Delta\left(Z_{S}, 2\right)$ the closed subspace of $\ell_{2}\left(Z_{S},\|\cdot\|_{S}^{n}\right)$ consisting of all the vectors of $\ell_{2}\left(Z_{S},\|\cdot\|_{S}^{n}\right)$ on the form $(x, x, x, \ldots)$.

We should note that, as for each $t \in S, e_{t} \in W_{S}$, we have $\left\|e_{t}\right\|_{S}^{n} \leqslant 2^{-n}$, and hence $\left(e_{t}, e_{t}, e_{t}, \ldots\right) \in \Delta\left(Z_{S}, 2\right)$. As $\left(e_{t}\right)_{t \in S}$ remains a suppression unconditional basis for $\left(Z_{S},\|\cdot\|_{S}^{n}\right)$ for each $n$, one also sees that it is a suppression unconditional basis for $\Delta\left(Z_{S}, 2\right)$. We shall denote by $\|\cdot\|_{S}$ the norm on $\left(e_{t}\right)_{t \in S}$ giving the space $\Delta\left(Z_{S}, 2\right)$. 
Proposition 29. Suppose $\phi: S \rightarrow T$ is an embedding of pruned subtrees of $\mathrm{T}$ satisfying $\phi(u, s)=\left(u, s^{\prime}\right)$, i.e., $\phi$ preserves the first coordinate of every element of S. Then

$$
M_{\phi}: e_{(u, s)} \mapsto e_{\phi(u, s)}
$$

extends to a linear isometry from $\Delta\left(Z_{S}, 2\right)$ into $\Delta\left(Z_{T}, 2\right)$. Moreover, $M_{\phi}\left(\Delta\left(Z_{S}, 2\right)\right)$ is 1-complemented in $\Delta\left(Z_{T}, 2\right)$.

We now need the following fundamental result of Argyros and Dodos on the structure of the spaces $\Delta\left(Z_{S}, 2\right)$. We shall formulate their result only for the special case of the spaces that we construct here, which are particular examples of the more general construction in [1, and only mention the aspects we need.

Theorem 30 (S. Argyros and P. Dodos 1, Theorem 71, Theorem 74). Let $S$ be a pruned subtree of the complete tree on $2 \times \omega, \mathrm{T}$. For each $\sigma \in[S]$, denote by $\mathcal{X}_{\sigma}$ the closed subspace of $\Delta\left(Z_{S}, 2\right)$ spanned by the sequence $\left(e_{t}\right)_{t \subseteq \sigma}$ and by $P_{\sigma}$ the (norm 1) projection of $\Delta\left(Z_{S}, 2\right)$ onto $\mathcal{X}_{\sigma}$.

(1) For each $\sigma \in[S], \mathcal{X}_{\sigma}$ is isomorphic to $X_{\sigma} \subseteq Z_{S}$.

(2) If $Y \subseteq \Delta\left(Z_{S}, 2\right)$ is an infinite-dimensional closed subspace such that for all closed infinite-dimensional subspaces $Z \subseteq Y$ and $\sigma \in[S]$ the projection $P_{\sigma}: Z \rightarrow \mathcal{X}_{\sigma}$ is not an isomorphic embedding (in this case we say that $Y$ is $Z_{S}$-singular), then $Y$ contains $\ell_{2}$.

Moreover, $\Delta\left(Z_{S}, 2\right)$ is reflexive.

We are now ready to prove the main result of this article.

Theorem 31. The relation of isomorphism between separable Banach spaces is a complete analytic equivalence relation.

Our proof will at the same time also show the following two results

Theorem 32. The relation of Lipschitz isomorphism between separable Banach spaces is a complete analytic equivalence relation.

and

Theorem 33. The relations of embeddability, complemented embeddability and Lipschitz embeddability between separable Banach spaces are complete analytic quasiorders.

For good order, we should mention that by Theorem 32 the first problem of 20] is answered. Theorem 32 should also be contrasted with the result in 20] stating that the relation of Lipschitz isomorphism between compact metric spaces is Borel bireducible with a complete $\boldsymbol{K}_{\sigma}$-equivalence relation. Thus Lipschitz isomorphism between compact metric spaces has the same complexity as equivalence between Schauder bases, while between separable Banach spaces it has the same complexity as permutative equivalence.

Proof. The map that will simultaneously take care of all the reductions is the obvious one

$$
S \mapsto \Delta\left(Z_{S}, 2\right)
$$

for all pruned normal subtrees $S$ of T.

We thus only need to notice the properties of this map. First of all, if $S$ and $T$ are two pruned normal trees such that $S \leqslant \boldsymbol{\Sigma}_{1}^{1} T$ as witnessed by some $\beta \in \omega^{\omega}$, then we 
can define an embedding $\phi: S \rightarrow T$ by $\phi(u, s)=\left(u, s+\left.\beta\right|_{|s|}\right)$. By Proposition 29] the map $M_{\phi}: \Delta\left(Z_{S}, 2\right) \rightarrow \Delta\left(Z_{T}, 2\right)$ is an isomorphic embedding, which moreover is a permutative equivalence between $\left(e_{t}\right)_{t \in S}$ and a subsequence of $\left(e_{t}\right)_{t \in T}$. So, in particular, if $S \equiv_{\boldsymbol{\Sigma}_{1}^{1}} T$, then $\left(e_{t}\right)_{t \in S}$ and $\left(e_{t}\right)_{t \in T}$ are permutatively equivalent with subsequences of each other and hence, as they are both unconditional, they are permutatively equivalent and thus $\Delta\left(Z_{S}, 2\right)$ and $\Delta\left(Z_{T}, 2\right)$ are isomorphic.

On the other hand, if $S \Phi_{\boldsymbol{\Sigma}_{1}^{1}} T$, we find an $\alpha \in \operatorname{proj}[S] \backslash \operatorname{proj}[T]$ and a $\beta$ such that $\sigma=(\alpha, \beta) \in[S]$. We thus notice that $\ell_{p_{\alpha}} \cong X_{\sigma} \cong \mathcal{X}_{\sigma}$. We claim that $\Delta\left(Z_{T}, 2\right)$ contains no subspace isomorphic to $\ell_{p_{\alpha}}$. For if $Y$ is any subspace of $\Delta\left(Z_{T}, 2\right)$, then either $Y$ is $Z_{T}$-singular, in which case, $Y$ contains a copy of $\ell_{2}$ and hence is not isomorphic to $\ell_{p_{\alpha}}$, or there is a subspace $Z \subseteq Y$ and a branch $\rho=(\gamma, \delta) \in[T]$ such that $P_{\rho}: Z \rightarrow \mathcal{X}_{\rho}$ is an isomorphic embedding. But then $Z$ is isomorphic to a subspace of $\mathcal{X}_{\rho} \cong \ell_{p_{\rho}}$ and hence contains a subspace isomorphic to $\ell_{p_{\rho}}$. As $\rho \neq \alpha$, $Y$ cannot be isomorphic to $\ell_{p_{\alpha}}$, and thus, finally, $\Delta\left(Z_{S}, 2\right)$ does not embed into $\Delta\left(Z_{T}, 2\right)$. Since by Theorem $30 \Delta\left(Z_{S}, 2\right)$ is reflexive, it follows that $\Delta\left(Z_{S}, 2\right)$ does not Lipschitz embed into $\Delta\left(Z_{T}, 2\right)$ (see [3] chapter 7 for more on this).

This shows that $\left(\leqslant_{\boldsymbol{\Sigma}_{1}^{1}}, \mathbb{E}_{\boldsymbol{\Sigma}_{1}^{1}}\right)$ reduces to the couple (complemented isomorphic embeddability, non-Lipschitz embeddability) between separable Banach spaces and thus the relations of complemented embeddability, embeddability, and Lipschitz embeddability are analytic complete. Similarly, $\left(\equiv_{\boldsymbol{\Sigma}_{1}^{1}}, \neq_{\boldsymbol{\Sigma}_{1}^{1}}\right)$ reduces to the relations of isomorphism and Lipschitz isomorphism and these are analytic complete too.

Corollary 34. The relations of topological embeddability and topological isomorphism between Polish groups are complete analytic as quasiorders and equivalence relations respectively.

Before we prove this, let us first define the space of Polish groups, $\mathfrak{G}$, as the Effros-Borel space of closed subgroups of $\operatorname{Hom}\left([0,1]^{\mathbb{N}}\right)$. By a result of Uspenskiu 24], this group contains all other Polish groups as closed subgroups up to topological isomorphism. Two Polish groups are said to be topologically isomorphic if there is a continuous group isomorphism between them. Such an isomorphism is automatically a homeomorphism and thus an isomorphism of the corresponding uniform structures. Similarly, one Polish group is topologically embeddable into another if it is topologically isomorphic with a closed subgroup.

Proof. Notice that if $\phi: X \rightarrow Y$ is a topological isomorphism of two Banach spaces considered as Polish groups, then, in particular, $\phi$ is an isomorphism of $X$ and $Y$ as $\mathbb{Q}$-vector spaces (since it preserves divisibility). But any continuous $\mathbb{Q}$-vector space isomorphism between two Banach spaces is also a linear isomorphism. The same argument applies to embeddings. So group isomorphism/embedding coincides with linear isomorphism/embedding.

\section{REFERENCES}

[1] S. A. Argyros and P. Dodos, Genericity and amalgamation of classes of Banach spaces, preprint (2005).

[2] H. Becker and A. S. Kechris, The descriptive set theory of Polish group actions, London Mathematical Society Lecture Note Series, 232. Cambridge University Press, Cambridge, 1996. xii+136 pp. 
[3] Y. Benyamini and J. Lindenstrauss, Geometric nonlinear functional analysis. Vol. 1, Amer. Math. Soc., Providence, RI, 2000.

[4] B. Bossard, A coding of separable Banach spaces. Analytic and coanalytic families of Banach spaces. Fund. Math. 172 (2002), no. 2, 117-152.

[5] R. Camerlo, Universality of embeddability relations for coloured total orders, Order 22 (2005), no. 3, 289-300 (2006).

[6] W.J. Davis, T. Figiel, W.B. Johnson, and A. Pełczyński, Factoring weakly compact operators, J. Funct. Anal., 17 (1974), 311-327.

[7] V. Ferenczi, On the number of pairwise permutatively inequivalent basic sequences in a Banach space, J. Funct. Anal., to appear.

[8] V. Ferenczi and E. M. Galego, Some equivalence relations which are Borel reducible to isomorphism between separable Banach spaces, Israel J. Math., 152(2006), 61-82.

[9] V. Ferenczi and C. Rosendal, On the number of non-isomorphic subspaces of a Banach space, Studia Math. 168 (2005), no. 3, 203-216.

[10] H. Friedman and L. Stanley, A Borel reducibility theory for classes of countable structures, J. Symb. Log. 54 (1989), 894-914.

[11] S. Gao and A. S. Kechris, On the classification of Polish metric spaces up to isometry, Mem. Amer. Math. Soc. Vol 161, (2003).

[12] W.T. Gowers and B. Maurey, The unconditional basic sequence problem, J. Amer. Math. Soc. 6 (1993), 851-874.

[13] A. Kechris and A. Louveau, The classification of hypersmooth Borel equivalence relations, J. Amer. Math. Soc., Vol. 10, no. 1, (1997).

[14] A. Louveau and C. Rosendal, Complete analytic equivalence relations, Trans. Amer. Math. Soc. 357 (2005), 4839-4866.

[15] A. Marcone and C. Rosendal, The complexity of continuous embeddability between dendrites, J. Symb. Log. 69 (2004), 663-673.

[16] J. Melleray, Computing the complexity of the relation of isometry between separable Banach spaces, eprint: arXiv:math.FA/0511456.

[17] B.S. Mityagin, Equivalence of bases in Hilbert scales, (Russian) Studia Math. 37 (1970), 111-137.

[18] A. Pełczyński, Universal bases, Studia Math. 32 (1969), 247-268.

[19] C. Rosendal, Etude descriptive de l'isomorphisme dans la classe des espaces de Banach, Thèse de Doctorat de l'Université Paris 6 (2003).

[20] C. Rosendal, Cofinal families of Borel equivalence relations and quasiorders, J. Symb. Log. 70 (2005), no. 4, 1325-1340.

[21] S. Thomas, personal communication.

[22] S. Thomas and B. Velickovic, On the complexity of the isomorphism relation of finitely generated groups, J. Algebra 217 (1999), no. 1, 352-373.

[23] B. S. Tsirelson, It is impossible to imbed $\ell_{p}$ or $c_{0}$ into an arbitrary Banach space, (Russian) Funktsional. Anal. i Priložen. 8 (1974), no. 2, 57-60.

[24] V. V. Uspenskiur, A universal topological group with a countable basis, (Russian) Funktsional. Anal. i Priložen. 20 (1986), no. 2, 86-87.

Address of V. Ferenczi and A. Louveau:

Équipe d'Analyse Fonctionnelle,

Université Pierre et Marie Curie - Paris 6,

Boite 186,

4, Place Jussieu,

75252 Paris Cedex 05, France.

ferenczi@ccr.jussieu.fr, louveau@ccr.jussieu.fr

Address of C. Rosendal:

Department of mathematics,

University of Illinois at Urbana-Champaign, 
273 Altgeld Hall, MC 382,

1409 W. Green Street,

Urbana, IL 61801, USA.

rosendal@math. uiuc.edu 